\title{
Detailed Uncertainty Analysis of the Ares I A106 Liftoff/Transition Database
}

\author{
Jeremy L. Hanke* \\ NASA Langley Research Center, Hampton, VA, 23681-2199
}

\begin{abstract}
The Ares I A106 Liftoff/Transition Force and Moment Aerodynamics Database describes the aerodynamics of the Ares I Crew Launch Vehicle (CLV) from the moment of liftoff through the transition from high to low total angles of attack at low subsonic Mach numbers. The database includes uncertainty estimates that were developed using a detailed uncertainty quantification procedure. The Ares I Aerodynamics Panel developed both the database and the uncertainties from wind tunnel test data acquired in the NASA Langley Research Center's 14- by 22-Foot Subsonic Wind Tunnel Test 591 using a 1.75 percent scale model of the Ares $I$ and the tower assembly. The uncertainty modeling contains three primary uncertainty sources: experimental uncertainty, database modeling uncertainty, and database query interpolation uncertainty. The final database and uncertainty model represent a significant improvement in the quality of the aerodynamic predictions for this regime of flight over the estimates previously used by the Ares Project. The maximum possible aerodynamic force pushing the vehicle towards the launch tower assembly in a dispersed case using this database saw a 40 percent reduction from the worst-case scenario in previously released data for Ares I.
\end{abstract}

\section{Nomenclature}

\begin{tabular}{|c|c|c|}
\hline \multicolumn{3}{|c|}{ Acronyms and Abbreviations } \\
\hline CLV & $=$ & crew launch vehicle \\
\hline $\mathrm{CP}$ & $=$ & center of pressure \\
\hline $\mathrm{CxP}$ & $=$ & Constellation Program \\
\hline $\mathrm{DAC}$ & $=$ & design analysis cycle \\
\hline DB & $=$ & database \\
\hline DBI & $=$ & database query interpolation \\
\hline DBM & $=$ & database modeling \\
\hline DOF & $=$ & degree(s) of freedom \\
\hline EXP & $=$ & experimental \\
\hline F\&M & & force $(\mathrm{s})$ and moment(s) \\
\hline FS & $=$ & first stage \\
\hline FTV & & flight test vehicle \\
\hline GN\&C & $=$ & Guidance, Navigation and Control \\
\hline GP & $=$ & gimbal point of the first stage \\
\hline LaRC & & Langley Research Center \\
\hline MLP & $=$ & mobile launch platform \\
\hline $\mathrm{MRC}$ & $=$ & moment reference center \\
\hline OML & $=$ & outer mold line \\
\hline RSS & $=$ & root-sum-square \\
\hline SBU & $=$ & sensitive but unclassified \\
\hline UQ & $=$ & uncertainty quantification \\
\hline UM & $=$ & uncertainty modeling \\
\hline UPWT & & Unitary Plan Wind Tunnel (NASA LaRC facility) \\
\hline WT & $=$ & wind tunnel \\
\hline $14 \times 22$ & $=$ & 14- by $22-$ Foot Subsonic Wind Tunnel (NASA LaRC facility) \\
\hline
\end{tabular}

${ }^{*}$ Research Aerospace Engineer, Configuration Aerodynamics Branch, MS 499, Member AIAA. 


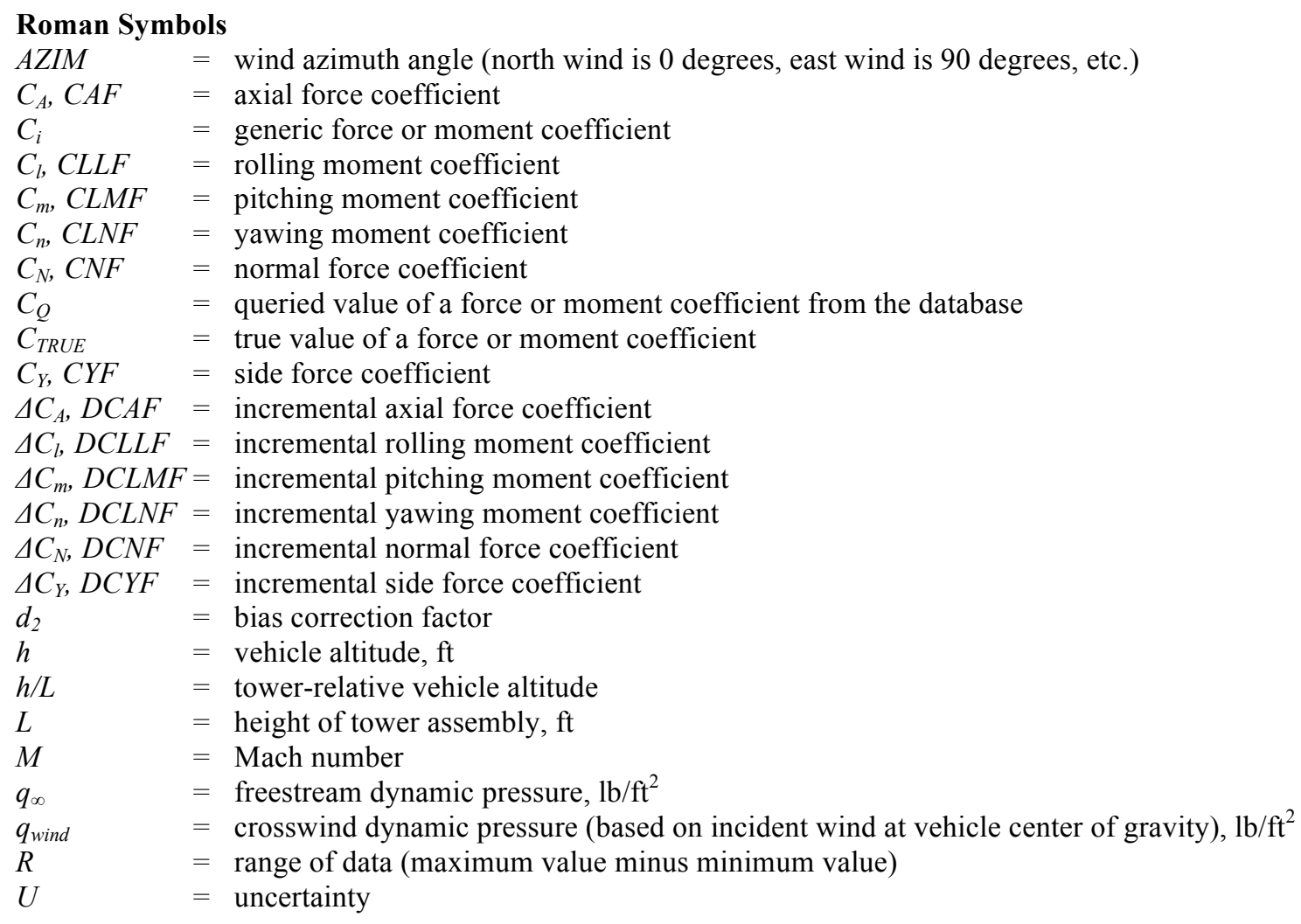

\section{Greek Symbols}

$\begin{array}{ll}\alpha & =\text { angle of attack } \\ \alpha_{T} & =\text { total angle of attack } \\ \beta & =\text { sideslip angle } \\ \Delta & =\text { denotes an incremental coefficient or value } \\ \phi_{a} & =\text { aerodynamic roll angle } \\ \rho_{\text {corr }} & =\text { correlation coefficient } \\ \sigma & =\text { standard deviation (or sample standard deviation) }\end{array}$

\section{Introduction}

$\mathrm{T}$ HE development and design process of the NASA crew launch vehicle (CLV), Ares I, required that the aerodynamics of the vehicles were adequately characterized for all phases of the flight envelope. Additionally, the Ares I Guidance, Navigation, and Control (GN\&C) team and other customers required estimates of uncertainties for each aerodynamic database (DB) for use in dispersion analyses and risk assessment. Two flight phases where aerodynamics significantly affected the GN\&C simulations were (1) the initial liftoff of the vehicle in the vicinity of the mobile launch platform (MLP) and launch tower assembly and (2) the transition from high to low total angle of attack flight at low subsonic Mach numbers $(\mathrm{M}<0.5)$. The Ares I A106 Lift-Off/Transition Force and Moment Aerodynamics Database provided the aerodynamic forces and moments on the CLV for these two flight phases for the Design Analysis Cycle 3 (DAC-3) vehicle based on the outer mold line (OML) designated A106. The overall DB was split into two segments: the Transition DB provided the freestream aerodynamics of the CLV for all angles of attack and sideslip angles, and the Tower Increments DB contained the incremental effect of the launch tower assembly on the CLV aerodynamics during liftoff. The Ares I Aerodynamics Panel team constructed this database entirely from experimental data obtained in NASA Langley Research Center's (LaRC) 14- by 22-Foot Subsonic Wind Tunnel $(14 \times 22)$ Test 591. For brevity, this document refers to the DB as a whole as the A106 Liftoff/Transition DB, and the wind tunnel test as 14x22 T591. Along with developing the nominal aerodynamic coefficients, the aero team conducted a detailed uncertainty quantification (UQ) analysis to estimate the 
uncertainties in the data and provide recommendations for the uncertainty modeling (UM) during dispersion analyses and risk assessment.

This document provides a summary of the detailed UQ methodology for the A106 Liftoff/Transition DB data, but the computation of each term in the UQ buildup is not described in detail. Reference 1 gives a more complete explanation of the details of the UQ process and contains the numerical data computed for all terms. This document provides several figures to illustrate key observations and graphically demonstrate the estimation and buildup of the final uncertainties. Some numerical quantities have been removed in the current paper to allow universal dissemination, since the Ares I CLV and its aerodynamics have been designated sensitive but unclassified (SBU). The focus of this report is on presenting the overall approach to uncertainty modeling for the database and showing the final uncertainties for the DB. Section II gives a short summary of the wind tunnel test and the database development. Section III presents the overall equations and general methodology used to compute the uncertainty. The general steps used to compute each individual component of the uncertainty are given in sections IV and V, and section VI describes the final results of the uncertainty model, as well as presenting some comparisons with previous data.

\section{A. The Ares I CLV}

Ares I was an in-line, two-stage, crew launch vehicle developed by NASA under the Constellation Project (CxP). The Ares I Aerodynamics Panel, spanning several NASA centers, conducted extensive aerodynamic testing and computational simulations in support of the overall vehicle integration effort. ${ }^{2-8}$ Figure 1 shows the OML of the A106 CLV along with the definitions of the body and missile axis systems, force and moment components, and flow angles. The A106 Liftoff/Transition DB reported all of the aerodynamic coefficients in the body axis system with the moment reference center (MRC) at the first stage (FS) gimbal point (GP), shown approximately as the origin of the axes systems in Fig. 1. However, the uncertainties were computed at the estimated center of pressure (CP) location $\left(X_{C P}\right)$, which is near the frustum of the CLV ( $\sim 13$ first stage diameters forward of the GP), and the implementation equations properly dispersed the uncertainties for use at any MRC.

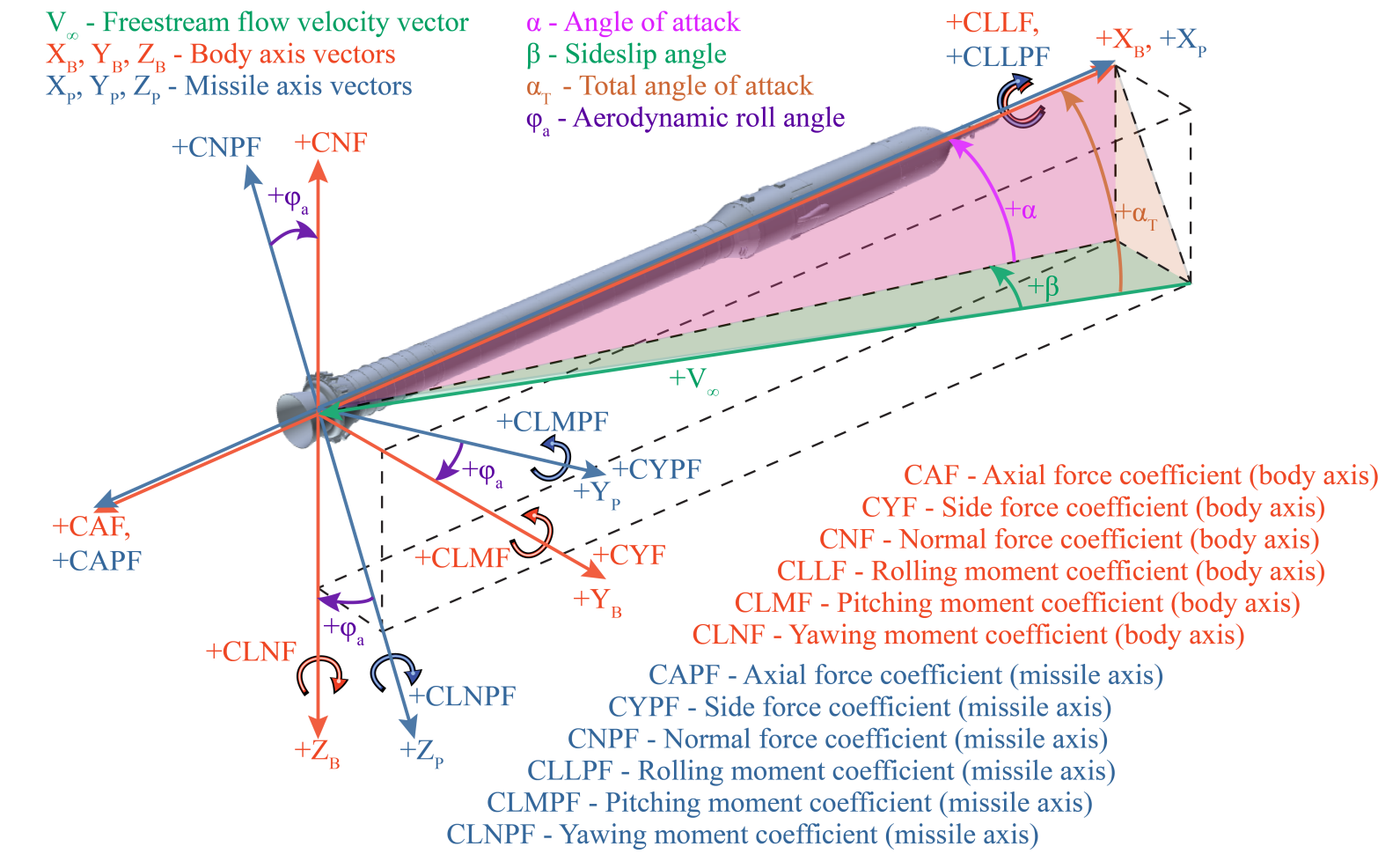

Figure 1. Definitions of body and missile axis systems, force and moment components, and flow angles for the Ares I CLV A106 OML. 


\section{Summary of Wind Tunnel Test and Database Development}

\section{A. 14x22 Test 591 Summary}

NASA LaRC $14 \times 22$ Test 591 was a force and moment wind tunnel test of a 1.75 percent scale Ares I model using the A106 geometry. Reference 9 provides details about the test and the data acquired. The objectives of the test were to measure the aerodynamic forces and moments on the Ares I CLV during the liftoff phase of the flight before the vehicle clears the tower assembly, as well as the transition from high to low angles of attack at low Mach numbers $(\mathrm{M}<0.3)$. The test consisted of three distinct phases:

1) A limited grit strategy investigation at the outset of the test (grit study phase),

2) Testing at total angles of attack from 0 to 90 degrees for the transition phase of flight (transition phase), and

3) Testing with the CLV at a total angle of attack of 90 degrees for all wind directions incident to the launch tower assembly (termed wind azimuth angle) and several tower-relative vehicle altitudes, both with and without the launch tower assembly (liftoff phase).

The time and resources available for this test did not allow for a full grit strategy investigation across the entire domain, which is a common challenge for high angle of attack testing. ${ }^{10-12}$ Instead, the grit study phase of $14 \times 22$ T591 sought to find a reasonable approach to use for the rest of the test based on available data and previous grit strategy best practices and to provide some information on the effect of the grit configuration on the data. The WT test team tested five different grit strategies during this phase, and the data from these configurations were used to quantify the grit uncertainty.

The transition phase of the test acquired data for the CLV model mounted on a mechanism that allowed testing for the entire range of total angle of attack (0-90 degrees) and aerodynamic roll angle (0-360 degrees). The model support structure used two fixed pitch-offset positions ( 0 and 45 degrees) of a variable pitch head mechanism to achieve the entire total angle of attack range. The test included pitch sweeps for both pitch-offset setups at constant aerodynamic roll angles in 15-degree increments from 0 to 360 degrees. Roll sweeps were also acquired at constant total angle of attack values throughout the domain.

For the liftoff phase of the test, the model assembly was mounted directly to the $14 \times 22$ vertical post, and a launch tower assembly model was installed with the CLV model to simulate the influence of the tower structure during liftoff. The launch tower assembly model for $14 \times 22$ T591 was a simplified design of the actual geometry in Ref. 13. The model retained the major features of the full-scale design, but small details of the tower geometry were omitted and the MLP was not modeled in detail. Figure 2 shows an isometric view of the tower assembly model design and the CLV model in proximity to the tower. The liftoff phase test setup allowed the test team to change the vertical position of the CLV model relative to the tower assembly model. This capability allowed for testing at various tower-relative vehicle altitudes $(h / L)$ from liftoff through tower clearance. The entire assembly could be rotated relative to the incoming flow to simulate all wind azimuth angles, which is the incident angle of the wind to the CLV and tower assembly as viewed from above (north wind is 0 degrees, east wind is 90 degrees, etc). Data for the CLV without the tower assembly model were acquired first to establish a baseline. Then, the team installed the tower assembly with the CLV model and acquired data for wind azimuth angles from 0 to 360 degrees and $h / L$ positions from 0.0 to 0.97 . Figure 3 illustrates definitions of the vehicle altitude and tower height, as well as a representative sketch of the incident winds just after liftoff with the freestream $\left(q_{\infty}\right)$ and crosswind dynamic pressures $\left(q_{\text {wind }}\right)$ identified. 


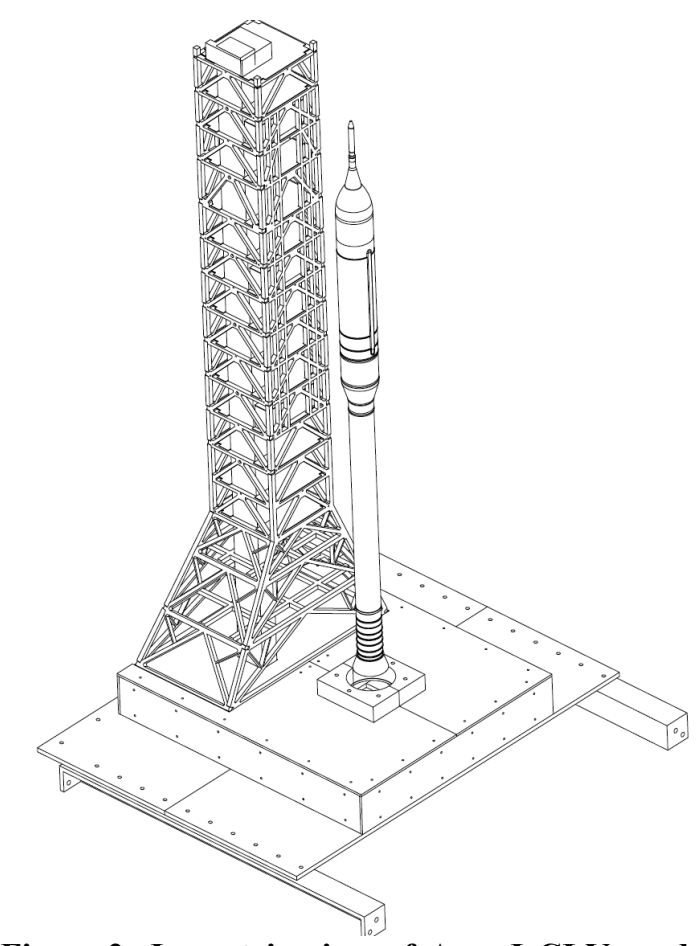

Figure 2. Isometric view of Ares I CLV model and Ares I tower assembly model geometries for 14x22 T591.

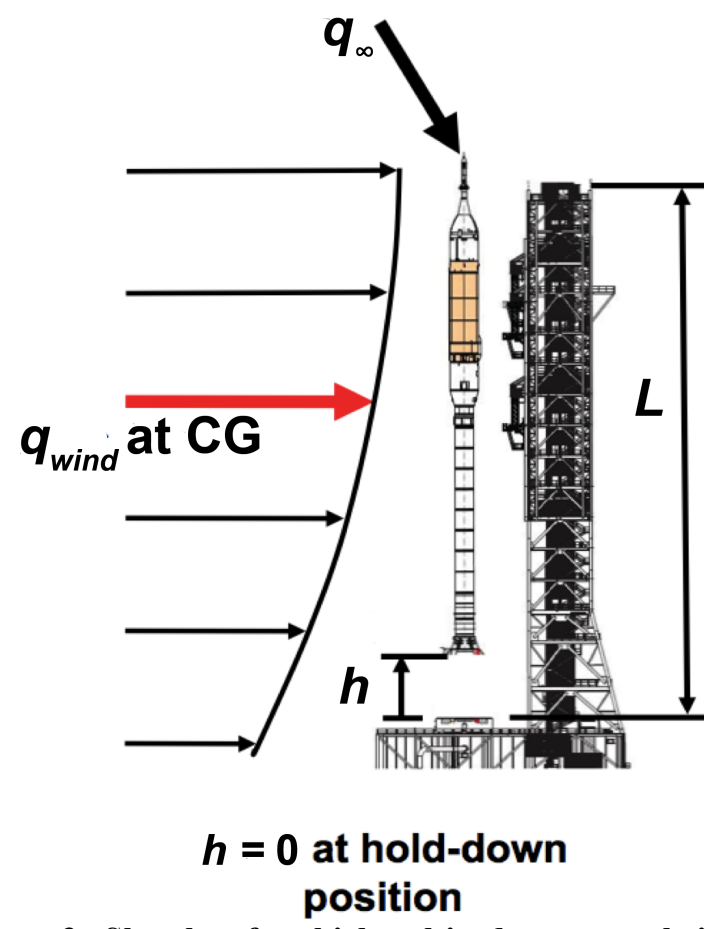

Figure 3. Sketch of vehicle altitude, tower height, freestream dynamic pressure, and crosswind dynamic pressure.

\section{B. A106 Liftoff/Transition Database Development}

The A106 Liftoff/Transition DB has two distinct parts: (1) the Transition DB accounts for the aerodynamics of the vehicle in the absence of the tower assembly for angles of attack and sideslip angles from -90 to 90 degrees, and (2) the Tower Increments DB reports the incremental effect of the tower assembly on the CLV. The details of the database development process and results are presented in Ref. 14.

The DB team developed the Transition DB primarily from the pitch sweeps in the transition phase of the $14 \times 22$ T591, but used the roll sweep data to fill any gaps in the pitch sweep data. Most of these pitch sweep gaps occurred at total angles of attack between 30 and 60 degrees because of significant model dynamics during testing. The final step in the Transition DB development was a linear interpolation from nominal WT total angle of attack and aerodynamic roll angle breakpoints to the final database breakpoints in angle of attack and sideslip angle. The resulting Transition DB response surface for the rolling moment coefficient $\left(C_{l}\right)$ exhibited unexpected, large fluctuations at low total angles of attack. The fluctuations were attributed to the balance being oversized relative to the rolling moments in the $14 \times 22 \mathrm{~T} 591$; the balance design load was 1000 in-lbs and was specially calibrated at 300 in-lbs for the test, but the maximum rolling moment measured was on the order of 1 or 2 in-lbs. The A106 Ascent Force and Moment Aerodynamics DB ${ }^{14}$ covers the nominal flight of the CLV from Mach 0.5 through first stage separation at low total angles of attack and used data from a WT test with a more sensitive balance in rolling moment relative to the measured loading than the $14 \times 22$ T591. Therefore, the team assumed that the A106 Ascent DB at Mach 0.5 provided a more reliable estimate of $C_{l}$ at Mach 0.3 than the $14 \times 22$ T591 data at low total angles of attack (less than 10 degrees). The $14 \times 22 \mathrm{~T} 591 C_{l}$ data at high total angles of attack (greater than 50 degrees) were merged with the A106 Ascent DB data at low total angles (less than 10 degrees) to construct the final $C_{l}$ database.

The Tower Increments DB was based on the data from wind azimuth angle sweeps of the baseline- and no-tower configurations in the liftoff phase of the $14 \times 22$ T591. The mean no-tower data were subtracted from the mean baseline-tower data at each wind azimuth angle and tower-relative vehicle altitude. Limitations in the turntable setup in the WT left a gap from 80 to 100 degrees in wind azimuth angle where data could not be acquired. The DB team made a symmetry assumption to populate the missing region with the data from 260 to 280 degrees. Data for the 
baseline tower configuration at $h / L$ of 0.97 revealed significant tower effects for that condition, so the team assumed that the tower incremental effects on the CLV extended to $h / L$ of 1.2 based on the observed trend data.

\section{General UQ Methodology}

\section{A. General Uncertainty Model for A106 Liftoff/Transition DB}

The UQ team developed the general methodology for uncertainty quantification and modeling for the A106 Liftoff/Transition DB as part of an overall effort to construct and apply a unified UQ methodology to all Ares I aerodynamic databases. The same general methodology used for the A106 Liftoff/Transition DB was also used for the A106 Ascent DB. ${ }^{15}$ In a general sense, the uncertainty interval for an aerodynamic coefficient queried from any database is intended to bound the true value of the coefficient at some level of confidence, as shown in Eq. (1).

$$
C_{Q}-U_{C, Q} \leq C_{T R U E} \leq C_{Q}+U_{C, Q}
$$

It is often not clear how to estimate $U_{C, Q}$ in Eq. (1) from the various sources of uncertainty. The A106 Liftoff/Transition DB was derived entirely from experimental data, and there are three identified sources of uncertainty in the queried database values:

1. Experimental uncertainty for the wind tunnel dataset used to build the database response surface,

2. Uncertainty in creating the database response surface from the experimental dataset, and

3. Uncertainty due to interpolation of the discrete database to an arbitrary query point.

This document refers to these terms as experimental (EXP) uncertainty, database modeling (DBM) uncertainty, and database query interpolation (DBI) uncertainty, respectively. The overall uncertainty for a queried value of the database is some combination of each uncertainty source. The errors from any source could be correlated with the errors from other sources, either positively or negatively. These correlations are often difficult to compute in practice; however, properly identifying correlation during uncertainty quantification can be vital. ${ }^{16}$ The uncertainty analysis for the A106 Liftoff/Transition DB assumed that each of the uncertainty sources were uncorrelated with the other sources, which simplifies the uncertainty model but may not be conservative if correlation exits. This assumption has been used traditionally for random uncertainty propagation equations ${ }^{17}$ and suggests combining the uncertainty sources using root-sum-square (RSS) equations. The general equation for the total uncertainty for the Transition DB was

$$
U_{C, Q}=\sqrt{U_{C, E X P}^{2}+U_{C, D B M}^{2}+U_{C, D B I}^{2}}
$$

The Tower Increments DB uncertainty model used the same methodology, though the uncertainty terms were estimated separately. The equation for the uncertainty in the Tower Increments DB was

$$
U_{\triangle C, Q}=\sqrt{U_{\triangle C, E X P}^{2}+U_{\triangle C, D B M}^{2}+U_{\triangle C, D B I}^{2}}
$$

The uncertainties for both segments of the A106 Liftoff/Transition DB are epistemic, not aleatory, because a single, unknown true value exists for each aerodynamic coefficient at every unique condition within the database. The reported uncertainties should be interpreted as intervals about the nominal database values, and the true values of the coefficients are assumed to lie somewhere within those intervals. There are no assumed probability distributions for these uncertainties; however, the Ares I GN\&C team dispersed the uncertainties with uniform distributions in their simulations.

\section{B. Methodology for Estimating Population Standard Deviations}

The UQ team used a common methodology to estimate the population standard deviations for various types of data variability for the A106 Liftoff/Transition DB; this procedure drew upon the methods outlined for the withintest repeatability analysis for the A106 Ascent DB. ${ }^{15}$ This procedure can be applied to any data set with more than one measurement of a system response quantity. If the underlying population of the data was a normal distribution, the standard deviation of the population would be estimated as follows:

1. Compute the range (maximum minus minimum value) of all available measurements at each point. 
2. Normalize (divide) the ranges by $d_{2}$, a statistical bias factor dependent only on the number of measurements available at each point. ${ }^{18}$ (The value of $d_{2}$ is 1.128 for 2 measurements, 1.693 for 3 measurements, etc.)

3. Pool the normalized ranges into representative subsets. Determine the subsets by examining the data in the entire domain, along with any information regarding the underlying physics, such as changes in test setup, physical regime boundaries, etc.

4. Compute the average of the normalized ranges in each subset, typically using either the mean or the median. The median is more robust to outliers. The average of the normalized ranges for the subset is an estimate of the population standard deviation, as shown in Eq. (4).

$$
\hat{\sigma}=\frac{\overline{R_{i}}}{d_{2}}
$$

The underlying assumption of normality can be tested by comparing the normalized ranges to an upper limit based on the estimated standard deviation; nearly all of the individual normalized ranges should fall below the upper limit. This methodology is commonly used in the Statistical Process Control (SPC) community. ${ }^{18}$ The upper limit used for the current UQ analysis was 3.5 times the estimated population standard deviation, a number based on the 0.995 critical point of the $R / \sigma$ distribution for two repeat measurements. ${ }^{19}$ About one out of every 200 normalized ranges would be expected to exceed this upper limit by chance if the population distribution was normal.

In practice, however, this is often not the case. Many sources of uncertainty in wind tunnel data such as repeatability can be approximated as normal distributions, though the actual distributions typically show heavier than expected tails (similar to a Student's t distribution). ${ }^{20}$ This behavior was noted in other Ares I wind tunnel tests. $^{21}$ To capture the effects of these "heavy tails" of the actual distribution, the UQ team modified the methodology to construct estimates of the population standard deviations. The modified methodology essentially reverses the normality test: an upper limit is computed based on the normalized range data, then the standard deviation is estimated from the upper limit. The modified procedure is as follows:

1. Compute the range of all measurements at each point.

2. Normalize the ranges by $d_{2}$.

3. Pool the normalized ranges into representative subsets.

4. Compute an upper limit that bounds a chosen percentage of the normalized ranges in a given subset. For this DB, the upper limit encompassed 99 percent of the normalized range data in each subset.

5. Estimate the population standard deviation by dividing the computed upper limit by 3.5 .

The UQ team used this procedure throughout the A106 Liftoff/Transition DB UQ analysis for estimating standard deviations of terms expected to have normal or near-normal population distributions. A critical step in the above methodology is pooling the data into intelligently chosen subsets. Typically, sets of data with similar test setups or physical regimes are pooled together. Some sets of data may need to be further subdivided into smaller pooling groups based on examination of the normalized range data. Figure 4 shows a representative normalized range plot for the Transition DB repeatability data and the estimated standard deviations and upper limits. In the figure, normalized range data for $C_{N}$ and $C_{Y}$ (as well as $C_{m}$ and $C_{n}$ ) were pooled together when computing the upper limits. The Ares I CLV is nearly axisymmetric, with the only exceptions being the protuberances on the vehicle, and the uncertainties $C_{N}$ and $C_{Y}$ (as well as $C_{m}$ and $C_{n}$ ) for an axisymmetric body would be expected to be roughly the same magnitude in the body axis system. Thus, the UQ analysis for this DB pooled these data for most terms to increase the degrees of freedom in each computation. 

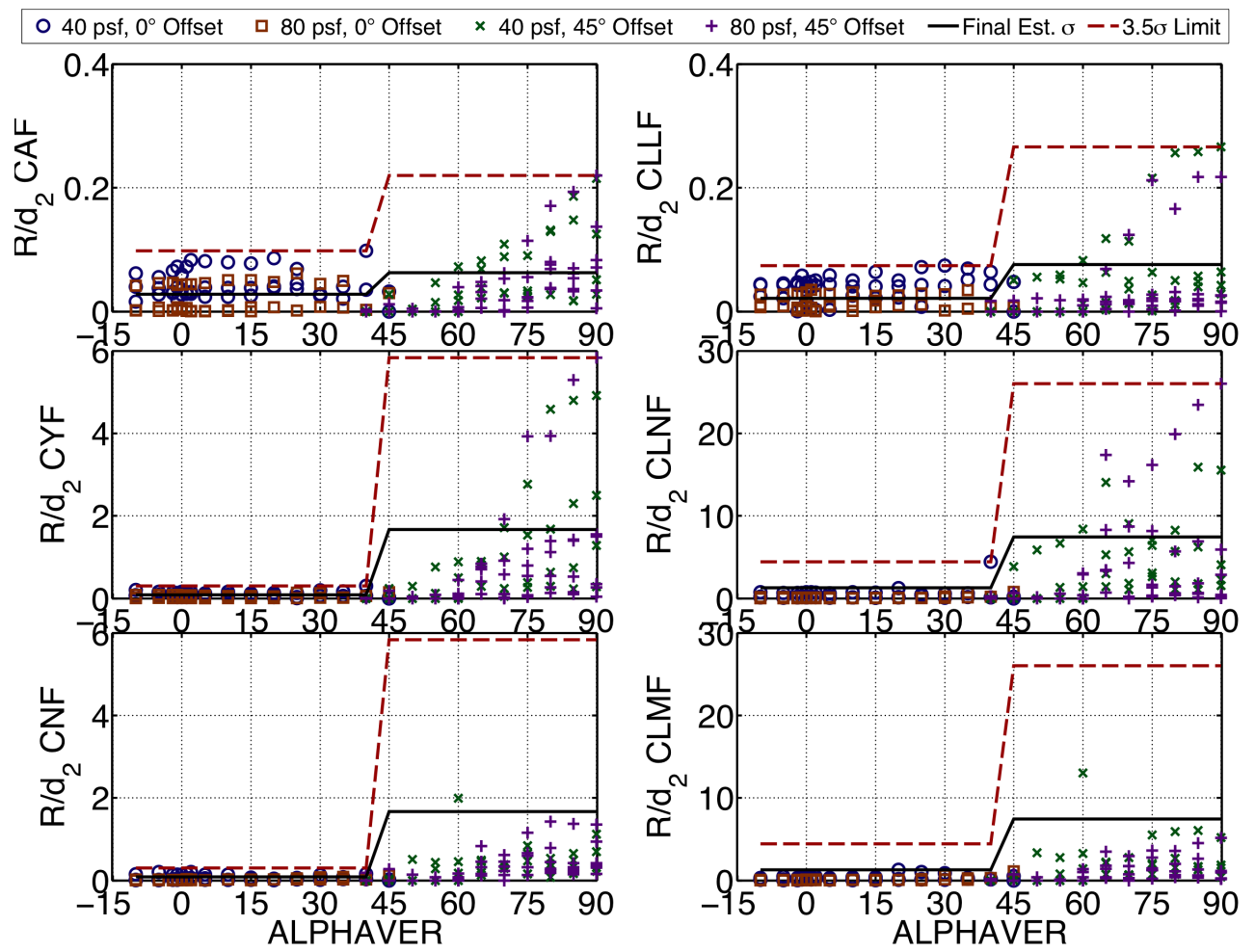

Figure 4. Transition DB normalized ranges for repeatability with the final repeatability estimates and upper limits versus $\alpha_{V}$.

\section{Uncertainty Estimates for the Transition DB}

\section{A. Experimental Uncertainty}

The most significant source of uncertainty for both segments of the A106 Liftoff/Transition DB was the experimental uncertainty. The experimental uncertainty for the Transition DB had five components:

1. Experimental data repeatability,

2. Experimental data reproducibility,

3. Grit effects uncertainty,

4. Balance calibration uncertainty, and

5. Base force correction uncertainty.

A brief summary of the analysis for each component is provided below. Each component was estimated separately, and the overall experimental uncertainty for the Transition DB was an RSS combination of the five components. Figure 5 shows a plot of the components of the experimental uncertainty for the Transition DB and the total RSS experimental uncertainty plotted versus total angle of attack. It is clear from Fig. 5 that the repeatability and reproducibility were significant contributors to the total experimental uncertainty for all coefficients. The grit effect uncertainty was significant for all coefficients except $C_{l}$. $C_{l}$ and $C_{A}$ were the only coefficients where the balance calibration uncertainty (denoted Balance Cal. in the figure) was a significant contributor. The base force correction uncertainty (denoted $C A B$ ) existed only for $C_{A}$ and was not a strong contributor to the total experimental uncertainty. 


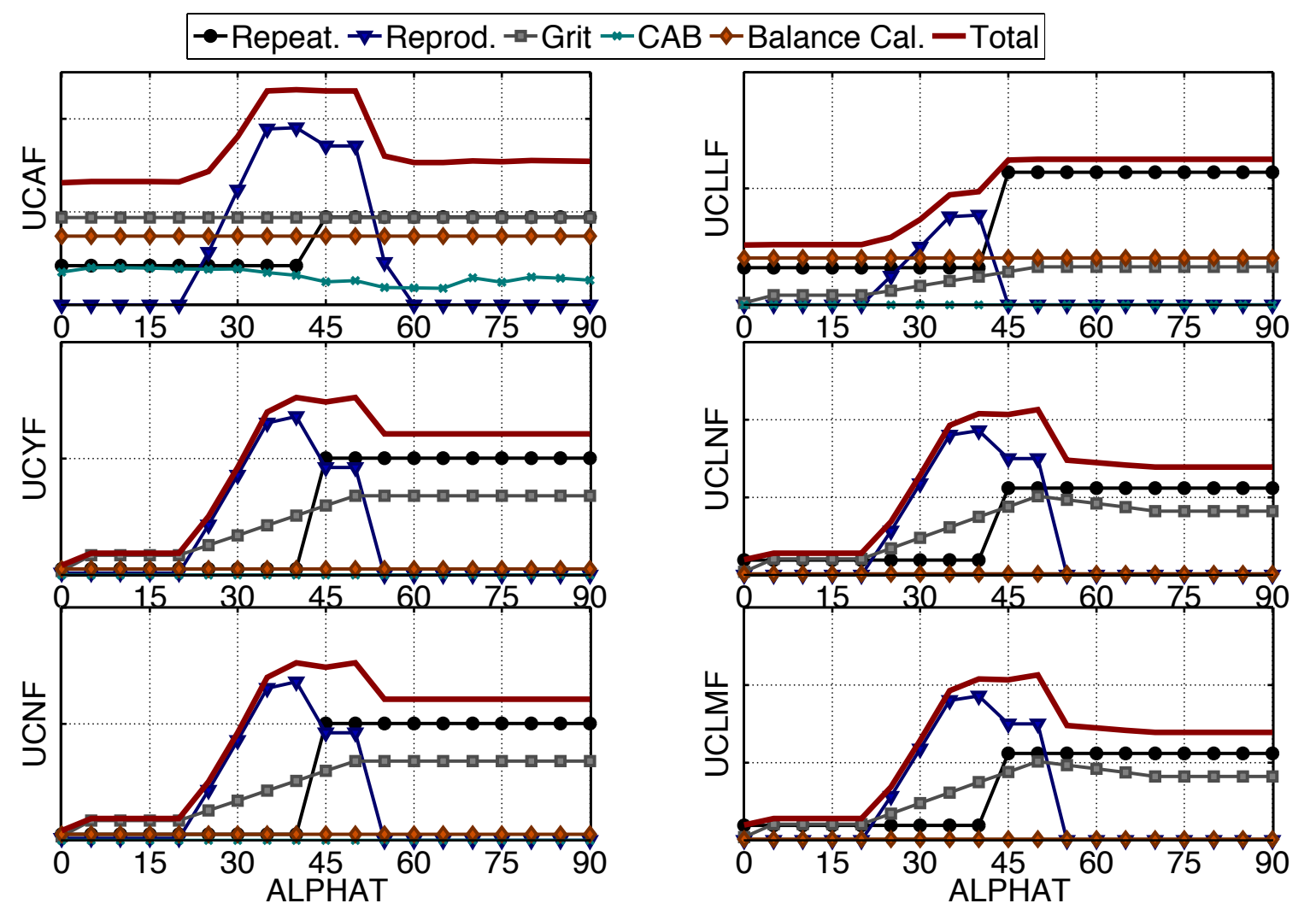

Figure 5. Experimental uncertainty components and total experimental uncertainties for Transition DB versus $\alpha_{T}$.

\section{Experimental Data Repeatability}

The experimental data repeatability measured the differences in data from two or more identical WT runs. The UQ team estimated the repeatability standard deviations using the methodology outlined above in Section III.B. The pitch sweep runs from the $14 \times 22$ T591 were the primary data source for the Transition DB, and only those runs were analyzed for repeatability. The normalized range data were pooled into separate subsets for the 0-degree and 45degree pitch offsets, and separate repeatability estimates were computed for each subset. Figure 4 shown earlier contained plots of the normalized range data and standard deviation estimates for the repeatability.

The UQ team propagated the estimated repeatability for the pitch runs in transition phase of the $14 \times 22 \mathrm{~T} 591$ into an experimental data uncertainty for the Transition DB. Since the methodology used to estimate the population standard deviations accounted for the effect of the "heavy tails," it is conservative to assume that the final uncertainties have normal distributions with the estimated population standard deviations. The Transition DB values were based on only one experimental measurement (mean of the baseline configuration data), and the experimental repeatability uncertainties for the Transition DB were set to equal three times the estimated standard deviations.

\section{Experimental Data Reproducibility}

In addition to identical replicate runs, data were acquired at the same conditions using differing test techniques during the transition phase of the $14 \times 22$ T591. In keeping with standard industry definitions, ${ }^{22}$ the team termed the variability in the data at these replicated conditions using different test setups as experimental reproducibility. The UQ team estimated the standard deviations for reproducibility using the methodology described in Section III.B. The estimated reproducibility standard deviations were then compared to the repeatability standard deviations from the exact replicate runs. If the reproducibility standard deviations were less than those for exact replicates, the reproducibility uncertainties were set to zero; otherwise, the reproducibility uncertainties only accounted for the additional variation beyond the repeatability, as shown in Eq. (5). The UQ team intended the uncertainty model to 
clearly denote the source of all uncertainties and thus did not combine the repeatability and reproducibility into a single term in the uncertainty model.

$$
\begin{array}{ll}
U_{E X P, \text { Reprod }}=3 \sqrt{\hat{\sigma}_{\text {Reprod }}^{2}-\hat{\sigma}_{\text {Repeat }}^{2}} & \text { for } \hat{\sigma}_{\text {Reprod }}>\hat{\sigma}_{\text {Repeat }} \\
U_{E X P, \text { Reprod }}=0 & \text { for } \hat{\sigma}_{\text {Reprod }} \leq \hat{\sigma}_{\text {Repeat }}
\end{array}
$$

The experimental data reproducibility for the Transition DB was based on 55 conditions replicated in both a pitch and a roll sweep and 65 replicated conditions from pitch sweep data where the two pitch offset setups overlapped. The UQ team computed separate standard deviation estimates for each situation and combined the results via RSS to estimate the overall reproducibility standard deviation, which was then compared to the repeatability standard deviation using Eq. (5) to compute the net reproducibility uncertainty term. Figure 5 showed that the reproducibility term was a significant contributor to the overall experimental uncertainty for total angles of attack from 20 to 55 degrees for all coefficients, but was insignificant for outside of that range.

\section{Grit Effects Uncertainty}

As mentioned earlier in Section II.A, the 14x22 T591 included only a limited grit study at the start of the test to determine a reasonable gritting approach. ${ }^{10}$ However, the baseline grit strategy used throughout the test may not have resulted in simulating the boundary layer transition behavior at the flight conditions as desired, so the uncertainty model contained a term to account for the possible error in gritting strategy. The grit strategy evaluation performed in the 14x22 T591 had many limitations that complicated the uncertainty analysis of the data. ${ }^{1}$ Because of these limitations, it was not possible to confidently estimate the errors between the baseline grit data and the best possible grit strategy data. Instead, the UQ team analyzed the data from all reasonable grit strategies tested and estimated that the grit effect uncertainty was equal to the maximum range from all of the grit configurations for each coefficient, pooling the data where reasonable. The estimated grit effect uncertainties were assumed to be symmetric about the nominal DB values with the true grit errors contained somewhere within the resulting interval.

No data were available below 50 degrees total angle of attack that could be used to evaluate the grit uncertainty. Instead, the UQ team used engineering judgment and historical research on similar bodies to estimate the grit effect uncertainties for that regime. Historical research on long, axisymmetric bodies similar to the Ares I CLV suggested that the aerodynamic behavior falls into several flow regimes. ${ }^{23}$ This information guided the grit uncertainty estimates for total angles of attack below 50 degrees. Unlike the other coefficients, the UQ team assumed that the grit effect uncertainty for $C_{A}$ was constant for all total angles of attack, since it is known that a uniform grit application (like the baseline grit strategy for 14x22 T591) causes an over-prediction of the axial force at low total angles of attack. Therefore, the grit effect errors in $C_{A}$ at low total angles of attack could be significant.

\section{Balance Calibration Uncertainty}

Many Ares I aerodynamic databases used test data on a CLV configuration with no protuberances (often called the clean configuration) to correct for systematic errors in the WT data. ${ }^{14}$ The $14 \times 22$ T591 did not include any runs for such a configuration, so this procedure could not be used to remove systematic biases in the wind tunnel data. One possible source of bias errors is the balance calibration curve-fit, so the uncertainty model included the balance calibration uncertainty. The balance calibration reports the standard errors of the curve-fit mismatches for a given calibration as 95 percent confidence intervals $(2 \sigma)$ in percent of full-scale load for each balance component. The author converted these values to aerodynamic coefficient uncertainties with 99.7 percent confidence $(3 \sigma)$ at a dynamic pressure of $40 \mathrm{psf}$. The uncertainties computed at $40 \mathrm{psf}$ are conservative for the Transition DB, since the lowest dynamic pressure for data used to develop the database was 40 psf. The design for the $14 \times 22$ T591 model assembly positioned the balance such that the balance axes aligned with the body axis system of the CLV model.

The balance calibration uncertainties were not significant contributors to the experimental uncertainties for most coefficients. The primary exception was the rolling moment, where the balance calibration uncertainty estimated using the above process was the same order of magnitude as the maximum $C_{l}$ magnitude measured during the $14 \times 22$ T591. The large $C_{l}$ balance calibration uncertainty greatly affected the GN\&C simulations of the CLV flight, so the aero team investigated whether the balance calibration uncertainty could be reduced for $C_{l}$. The team devised a new method for estimating possible balance calibration errors for $C_{l}$ after the GN\&C customers agreed to accept additional risk. The rolling moment for the CLV at a total angle of attack of 0 degrees would be expected to be small due to the near-axisymmetry of the CLV, so the variability in the $C_{l}$ data at 0 degrees measured in the $14 \times 22 \mathrm{~T} 591$ 
was used as an estimate of the possible balance calibration errors. The UQ team assumed that the maximum variability at 0 degrees was a reasonable estimate of the balance calibration curve-fit errors. This modification reduced the balance calibration uncertainty for $C_{l}$ by approximately 85 percent from the original value at 0 degrees.

\section{Base Force Correction Uncertainty}

The effects of pressures acting on the base and cavity of the model during the $14 \times 22$ T591 were removed using a base force correction. This correction only affected the axial force. Two pressure taps in the interior, or chamber, of the model measured the chamber pressure, and this pressure was assumed to apply to the base area of the model; however, the measured pressures appeared to be corrupted for some runs in the test. ${ }^{10}$ To address this, the mean base force correction from a set of runs where the pressure measurements appeared accurate was computed and used as the base force correction for all runs in the transition phase of the $14 \times 22$ T591. The uncertainty in the approximated correction was estimated as the range of the data pooled to compute the mean correction. No base force correction uncertainty was needed for coefficients other than $C_{A}$.

\section{B. Database Modeling Uncertainty}

The database modeling uncertainty term accounted for any errors generated in the process of creating the final database from the input data. For the Transition DB, the DBM uncertainty had three terms:

1. Uncertainty due to variability in the combined data set from dynamic pressures of 40 and $80 \mathrm{psf}$,

2. Uncertainty due to data variations with Mach/Reynolds number, and

3. Breakpoint interpolation uncertainty,

A brief summary of the analysis for each component is provided below. As with the experimental uncertainty, the UQ team assumed that all of these terms were uncorrelated and computed the total database modeling uncertainty using an RSS combination of the estimates for each term.

Figure 6 shows the components of the database modeling uncertainty along with the overall DBM uncertainties versus total angle of attack. The figure illustrates that the uncertainty due to pooling the data from two dynamic pressures (denoted QINF Pooling) was a significant contributor for all coefficients between 20 and 40 degrees. The breakpoint interpolation uncertainty was also significant, especially at the higher total angles of attack, and the Mach/Reynolds number variations term (denoted $M / R e$ ) was generally less significant than the other terms.

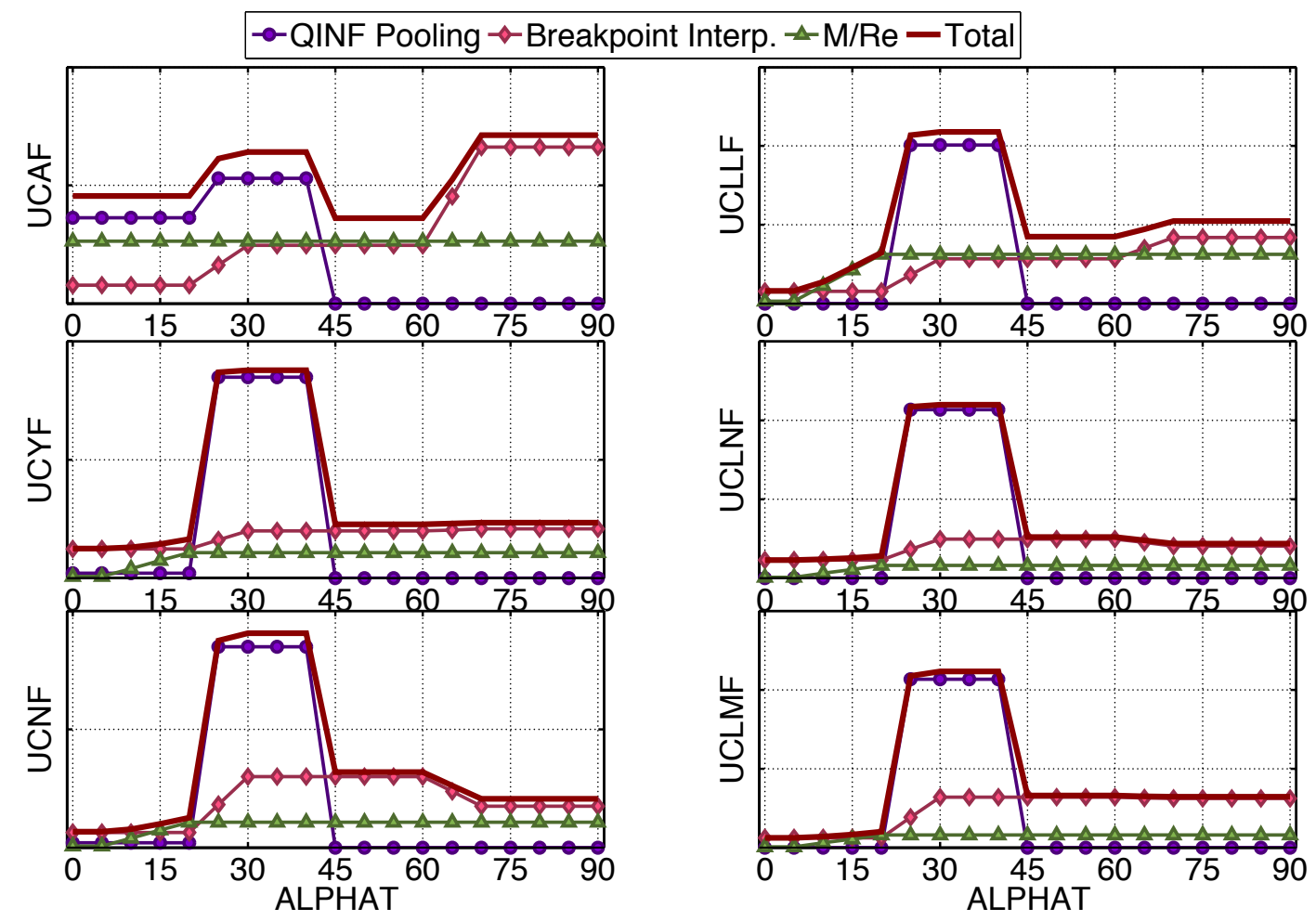

Figure 6. Database modeling uncertainty components and total database modeling uncertainties for Transition DB versus $\alpha_{T}$. 


\section{Variability in Combined Data from Dynamic Pressures of 40 and 80 psf}

The majority of the $14 \times 22$ T591 was conducted at two dynamic pressures: 40 and 80 psf. There were no significant qualitative differences between the results from the two dynamic pressures, as shown during the grit study at the beginning of the test. ${ }^{10}$ The DB team pooled the data from both dynamic pressures and used the mean data to develop the database. To test the assumption that data at both dynamic pressures were quantitatively similar, the UQ team quantified if the variability due to dynamic pressure was greater than the variability for identical repeats runs. First, the team pooled the data from both dynamic pressures as if the runs were replicate runs and used the methodology described in Section III.B to estimate the standard deviations for the combined set. These estimates were compared to the repeatability standard deviation estimates in the same way as for the reproducibility uncertainty, and any additional variability beyond the estimated repeatability was captured in the uncertainty due to pooling the dynamic pressure data. This uncertainty model placed this term under database modeling uncertainty instead of experimental uncertainty to clearly illustrate that pooling the data was a database development decision and does not reflect the accuracy of the experimental data. The results in Fig. 6 show that no additional variability was measurable for total angles of attack greater than 40 degrees, but a significant amount of variability beyond the estimated repeatability was observed between 20 and 40 degrees for all coefficients.

\section{Mach/Reynolds Number Variation Uncertainty}

The DB coefficients were assumed to be constant for all Mach numbers between 0.0 and 0.3 . Mach number, dynamic pressure, and Reynolds number cannot be varied independently in the $14 \times 22$, so the effects of Mach number on the WT data are confounded with the Reynolds number and dynamic pressure effects. The Mach numbers for runs at 40 and 80 psf were 0.165 and 0.236 , respectively. Dynamic pressure sweeps performed during the test varied dynamic pressure from 10 to $120 \mathrm{psf}(M=0.082$ to 0.292$)$. The Reynolds number also varied throughout the test along with Mach and dynamic pressure. The UQ team estimated an uncertainty for extending the data from Mach 0.165 and 0.236 to Mach 0.3 using the variations in the data from 80 to 120 psf in the dynamic pressure sweeps performed at 90 degrees total angle of attack. ${ }^{1}$ The UQ Team did not examine the uncertainty in extending the WT test data to Mach 0.0 but assumed that such errors were negligible.

\section{Breakpoint Interpolation Uncertainty}

The transition phase of the $14 \times 22$ T591 used pitch and roll sweeps to acquire data; therefore, the independent variables in each sweep were total angle of attack and aerodynamic roll angle, respectively. However, the GN\&C customers requested that the DB be provided using angle of attack and sideslip angle breakpoints regularly spaced throughout the domain. The DB team linearly interpolated the data from the wind tunnel breakpoints to the database breakpoints during the final step in the database development. Figure 7 shows the available wind tunnel data points and the final database breakpoints in the angle of attack and sideslip angle domain. Some WT points near the boundaries of the domain are not shown because of singularities in the trigonometric equations at a total angle of attack near 90 degrees. In reality, WT data exist that bound the entire database domain shown.

The UQ team estimated the error in this step of the DB development by using both a linear and cubic-spline interpolation to perform the same computation and analyzing the difference between the results. The team estimated the standard deviations for the breakpoint interpolation uncertainty using the methodology described above in Section III.B.

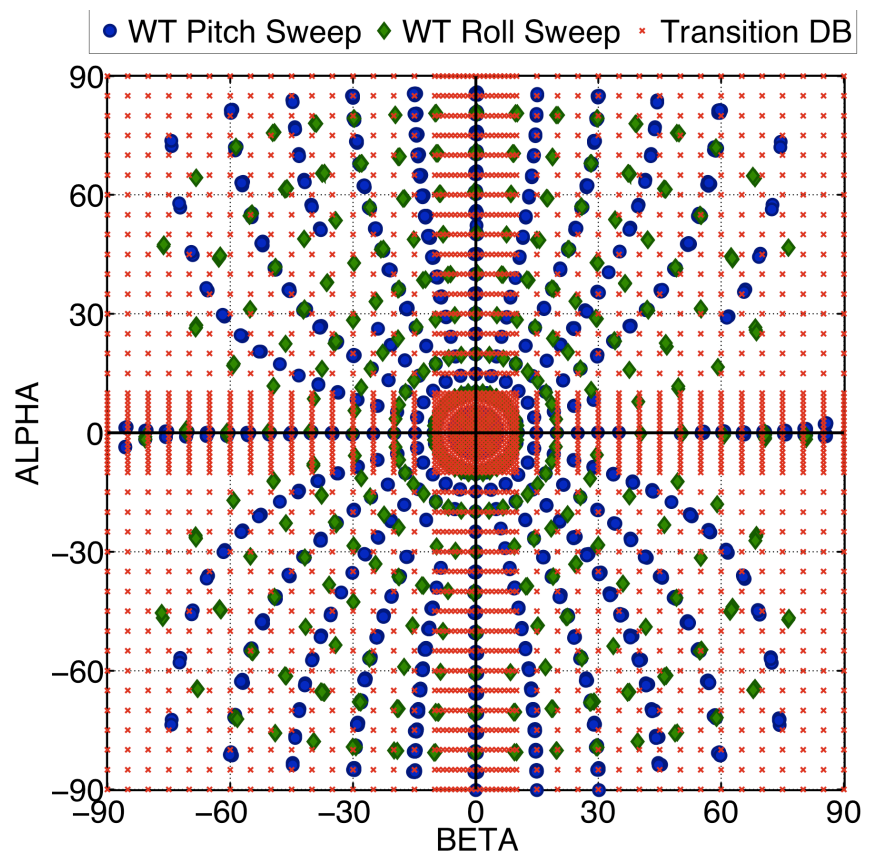

Figure 7. Comparison of WT and DB breakpoints for the Transition DB in the $\alpha$ and $\beta$ domain. 


\section{Database Query Interpolation Uncertainty}

The final source of uncertainty for the Transition DB is due to interpolation of the final discrete database to any arbitrary query point in the domain. Because the DB implementation specifies linear interpolation, the database query interpolation uncertainty is driven by the non-linearity of the DB response surface. The UQ team followed the same procedure used for computing the breakpoint interpolation uncertainty to estimate this uncertainty: interpolating the database to a finely spaced regular grid (every 0.5 degrees in both angle of attack and sideslip angle) using both linear and cubic spline interpolations and then analyzing the residuals between the two results. The standard deviations for the database query interpolation uncertainty were again estimated using the procedure described in Section III.B.

\section{Uncertainty Estimates for the Tower Increments DB}

The input data for the Tower Increments DB were acquired during the liftoff phase of the 14x22 T591. The same general UQ methodology used for the Transition DB was applied to the relevant data from the liftoff phase of the test to estimate the uncertainty terms for the Tower Increments DB. However, since some data were not available from the liftoff phase of testing, several of the uncertainty terms for the Tower Increments DB were actually derived from the Transition DB uncertainty results. Some other terms that were present in the Transition DB uncertainty model were omitted for the Tower Increments DB because of the incremental nature of the database.

\section{A. Experimental Uncertainty}

The UQ team built the experimental uncertainty for the Tower Increments DB from three components:

1. Experimental data repeatability,

2. Experimental data reproducibility, and

3. Grit effects uncertainty.

The following sections briefly describe the estimation of each of these terms. Due to the incremental nature of the DB, the UQ team assumed that any balance calibration curve-fit errors were removed. A base force correction uncertainty was not included for the Tower Increments DB because the base pressure data in the liftoff phase was not corrupted. ${ }^{10}$ The uncertainty model assumed that all of the experimental uncertainty terms were uncorrelated and the total experimental uncertainty was a RSS combination of the individual components. The experimental repeatability was a significant contributor for all other coefficients except $C_{A}$, and the grit effect uncertainty was significant for all coefficients except $C_{l}$ and $C_{A}$. The experimental data reproducibility uncertainty was negligible for all coefficients except $C_{A}$, where it was the dominant term in the experimental uncertainty. A single uncertainty was computed for each coefficient and applied to the entire Tower Increments DB domain since the available data did not suggest that any of the error sources varied with any independent variable.

\section{Experimental Data Repeatability}

There were only four conditions with identical replicate runs from the liftoff phase of the 14x22 T591: two wind azimuth sweeps each at 40 and $80 \mathrm{psf}$ for the baseline tower configuration. It is generally undesirable to estimate the experimental repeatability based on so few degrees of freedom. Therefore, the UQ team estimated the experimental data repeatability for the Tower Increments DB from the combined dynamic pressure data. The repeatability estimates from the combined dynamic pressure data set were similar in magnitude to the estimates from the four sets of identical replicates.

The standard deviations for repeatability were estimated by applying the methodology described in Section III.B to the combined dynamic pressure data set. The experimental repeatability uncertainty was again set equal to three times the estimated standard deviation, but since each value of the Tower Increments DB was constructed from two separate experimental terms (baseline- and no-tower data), the repeatability uncertainty for the DB was a RSS combination of the repeatability for each configuration. The final DB uncertainty equaled the square root of two times the estimated repeatability standard deviation, which was computed by pooling data from both configurations. The available data suggested that the repeatability for both configurations was similar.

\section{Experimental Data Reproducibility}

There were several conditions where data were acquired using differing test techniques during the liftoff phase of the $14 \times 22$ T591, and the UQ team analyzed the data variability at these conditions to estimate the experimental reproducibility. There were 34 conditions available for analysis where two or more types of sweep overlapped. As usual, the team estimated the reproducibility standard deviations for that data using the methodology described in Section III.B. The net reproducibility uncertainties again were computed by comparing the estimated reproducibility 
standard deviations to the repeatability estimates. After comparison to the repeatability, the reproducibility uncertainties were zero for all coefficients except axial force coefficient. The axial force reproducibility, however, ended up being the dominant term in the experimental uncertainty for $\Delta C_{A}$ in the Tower Increments DB. Large discrepancies between data from different sweeps at two of the 34 conditions drove the reproducibility uncertainty for $\Delta C_{A}$, but not enough data existed to determine if these conditions were outliers or caused by actual physical phenomenon. Again, the incremental nature of the Tower Increments DB resulted in an additional factor of the square root of two in the reproducibility uncertainty equation compared to Eq. (5) for the Transition DB.

\section{Grit Effects Uncertainty}

The 14x22 T591 did not use any alternate grit configurations during the liftoff phase. Without grit effect data from the liftoff phase of the test, the grit effects uncertainties for the Tower Increments DB were estimated using the uncertainties for the Transition DB. Standard error propagation methodology ${ }^{24}$ suggests that the level of correlation in the systematic grit error for both the baseline- and no-tower configurations from the liftoff phase of the $14 \times 22$ T591 can greatly affect the grit uncertainty for the Tower Increments DB. The UQ team assumed that the uncertainty magnitudes for both configurations were identical and equal to the grit uncertainty for the Transition DB at a total angle of attack of 90 degrees. The team believed that the grit effects on the two configurations were highly correlated, but the tower assembly may have affected the flow around the model so the team did not assume perfect correlation, which would have eliminated the grit uncertainty entirely. With no information to guide the choice of the correlation coefficient, the UQ team assumed a correlation coefficient of 0.8 and computed the final Tower Increments DB grit uncertainty accordingly.

\section{B. Database Modeling Uncertainty}

The basic structure and methodology for the database modeling uncertainty for the Tower Increments DB were the same as for the Transition DB. Unlike the Transition DB, however, the only term that was non-zero was the Mach/Reynolds number variation uncertainty. Therefore, the total database modeling uncertainty for the Tower Increments DB equaled the Mach/Reynolds number variation uncertainty.

\section{Variability in Combined Data from Dynamic Pressures of 40 and 80 psf}

As noted above in the experimental repeatability discussion, the data repeatability estimation pooled the data from both dynamic pressures. Thus, the experimental data repeatability uncertainty already captured any additional variability in the combined data set above the variability in exact replicate runs, and the DBM uncertainty term for pooling the dynamic pressure data equaled zero.

\section{Mach/Reynolds Number Variation Uncertainty}

No data were available for Mach numbers greater than $0.236\left(q_{\infty}=80 \mathrm{psf}\right)$ from the liftoff phase of the $14 \times 22$ T591, so the team could not directly measure the effect of extending the data from Mach 0.236 to 0.3 for the Tower Increments DB. Instead, the team used the same error propagation methodology and correlation assumptions (correlation coefficient of 0.8) used to estimate the grit effects uncertainty for the Tower Increments DB. Again, the magnitude of the Mach/Reynolds number variation uncertainties for each configuration were assumed to equal the Transition DB values at a total angle of attack of 90 degrees.

\section{Breakpoint Interpolation Uncertainty}

The breakpoints for the Tower Increments DB independent variables (wind azimuth angle and $h / L$ ) were identical to the nominal WT breakpoints, unlike in the Transition DB. Thus, the WT data were only interpolated from the actual conditions tested to the nominal conditions. Typically, the difference between the actual and nominal conditions was less than 0.1 degrees in wind azimuth angle and less than 0.01 in $h / L$. This interpolation was negligible, so the breakpoint interpolation uncertainties were zero.

\section{Database Query Interpolation Uncertainty}

The final source of uncertainty for the Tower Increments DB was the database query interpolation uncertainty. Again, the DB specifies linear interpolation to query the database response surface at any point in the domain. The same procedure was used to estimate this term as for the Transition DB. The finely spaced grid had breakpoints at every 1-degree in wind azimuth angle and every 0.05 in tower-relative vehicle altitude. 


\section{Final Results and Comparison to Previous Data}

The total uncertainties for the Transition DB and Tower Increments DB were a combination of the results for all of the component terms, given by Eq. (2) and Eq. (3), respectively. The uncertainties for the Transition DB and the Tower Increments DB were assumed to be independent and need to be dispersed separately during simulations. The following sections show the buildup of the total uncertainty for both segments of the DB and compare the results to previous data for this regime.

\section{A. Total Uncertainty for Transition DB}

\section{Total Uncertainty Buildup for Transition DB}

Figure 8 shows a plot of the three components of the uncertainty (experimental, database modeling, and database query interpolation) for the Transition DB and the total uncertainty versus total angle of attack. As expected, the database query interpolation uncertainty ( $U D B I$ in the figure) was relatively insignificant compared to the other two uncertainty sources. The experimental uncertainty $(U E X P)$ was the most significant component for all coefficients, especially at total angles of attack greater than 30 degrees. The database modeling uncertainty (UDBM) was only significant for $C_{Y}, C_{N}, C_{m}$, and $C_{n}$ below 40 degrees total angle of attack, where the additional variability due to pooling the data from dynamic pressures of 40 and 80 psf was the driving term in the database modeling uncertainty.
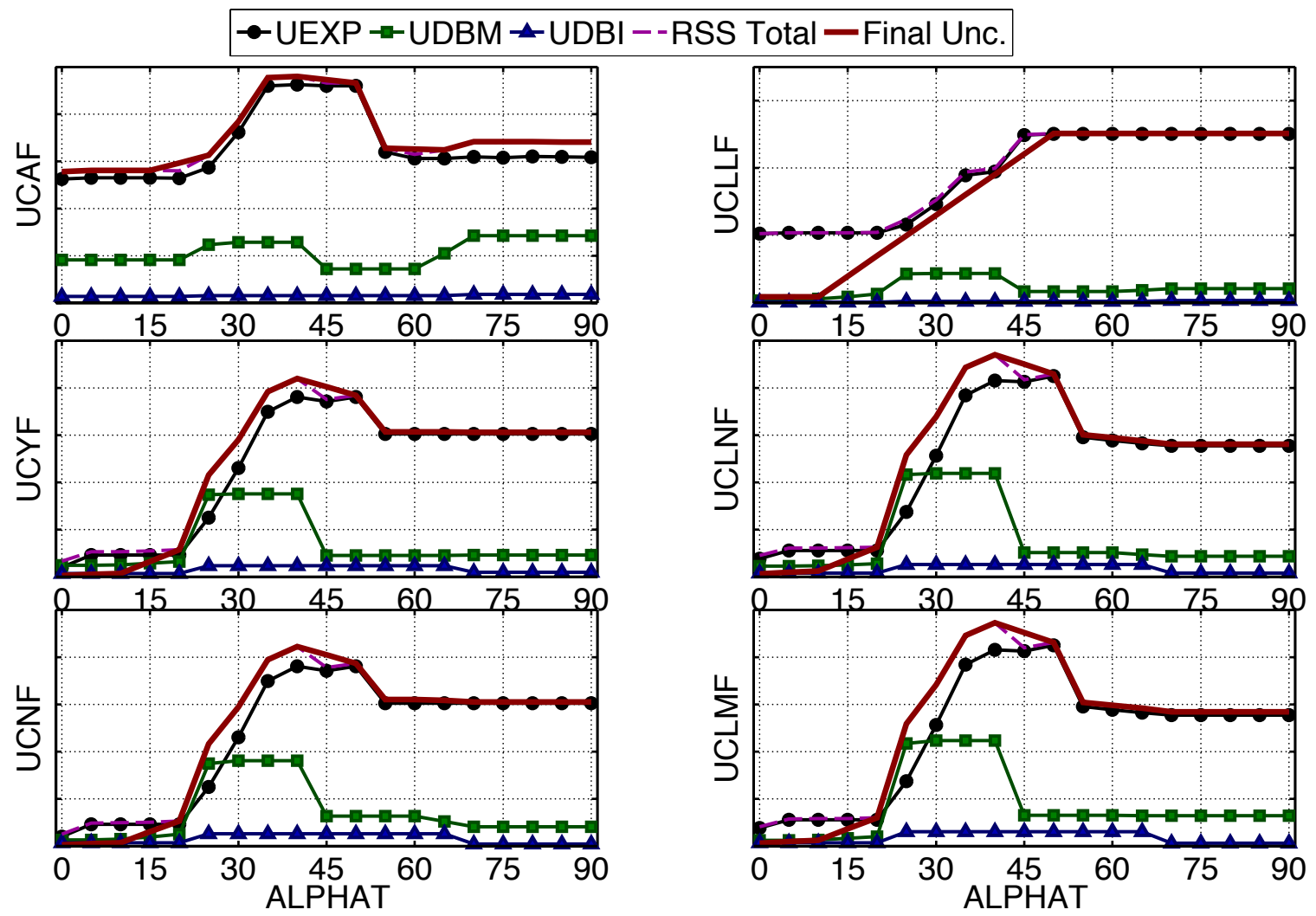

Figure 8. Transition DB total uncertainty buildup and final uncertainties versus $\alpha_{T}$.

The UQ team made several adjustments to the total uncertainties computed using Eq. (2) in the R1.1 update of the DB. These changes can be seen in Fig. 8 by noting the difference between the RSS Total curves, which represent the total uncertainty computed using Eq. (2), and the Final Unc. lines, which denote the final uncertainties. The majority of these adjustments were made because GN\&C feedback suggested that discontinuities between the uncertainties in the A106 Liftoff/Transition DB and the A106 Ascent DB affected the simulation results. The computed uncertainties for the Transition DB at low total angles of attack were much larger than the uncertainties from the A106 Ascent DB at Mach 0.5. In GN\&C dispersion simulations, such a discontinuity in the uncertainties created a corresponding jump in the dispersed coefficient values at the switchover point between the two databases. This was especially noticeable for the rolling moment, where the DB nominal values as well as the uncertainties were adjusted in the R1.1 update. 
The Ares aero team investigated the dominant sources of uncertainty for the Transition DB and decided that the computed uncertainties may have been overly conservative at low total angles of attack. Therefore, the final uncertainties for all Transition DB coefficients except $C_{A}$ equal the A106 Ascent DB uncertainties for those coefficients for total angles of attack from 0 to 10 degrees. Linear interpolation was used between the low total angle of attack uncertainties and the computed uncertainty values at higher angles. (Feedback from the GN\&C customers suggested that discontinuities in $C_{A}$ did not affect the simulations, so no adjustments were made.) As a final minor adjustment, the computed final uncertainties were adjusted slightly to remove any valleys in the uncertainty curves versus total angle of attack.

\section{Transition $D B$ and Uncertainty Visualization}

It is useful to visualize the magnitude of the uncertainties relative to the database. However, it can be difficult to do so for response surfaces in two or more dimensions. Instead, Fig. 9 shows the database and maximum uncertainty bounds for a slice of the overall response surface; in this case, the data are plotted versus total angle of attack for an aerodynamic roll angle of 0 degrees. The coefficients in the figure use the MRC of the DB (FS GP) for $C_{m}$ and $C_{n}$, and the uncertainties for $C_{m}$ and $C_{n}$ were properly transferred to the GP using the implementation equations. The plots show that the uncertainties are small relative to the DB at low total angles of attack for all coefficients except $C_{A}$, and that the uncertainties are most significant compared to the DB values between 30 and 60 degrees.

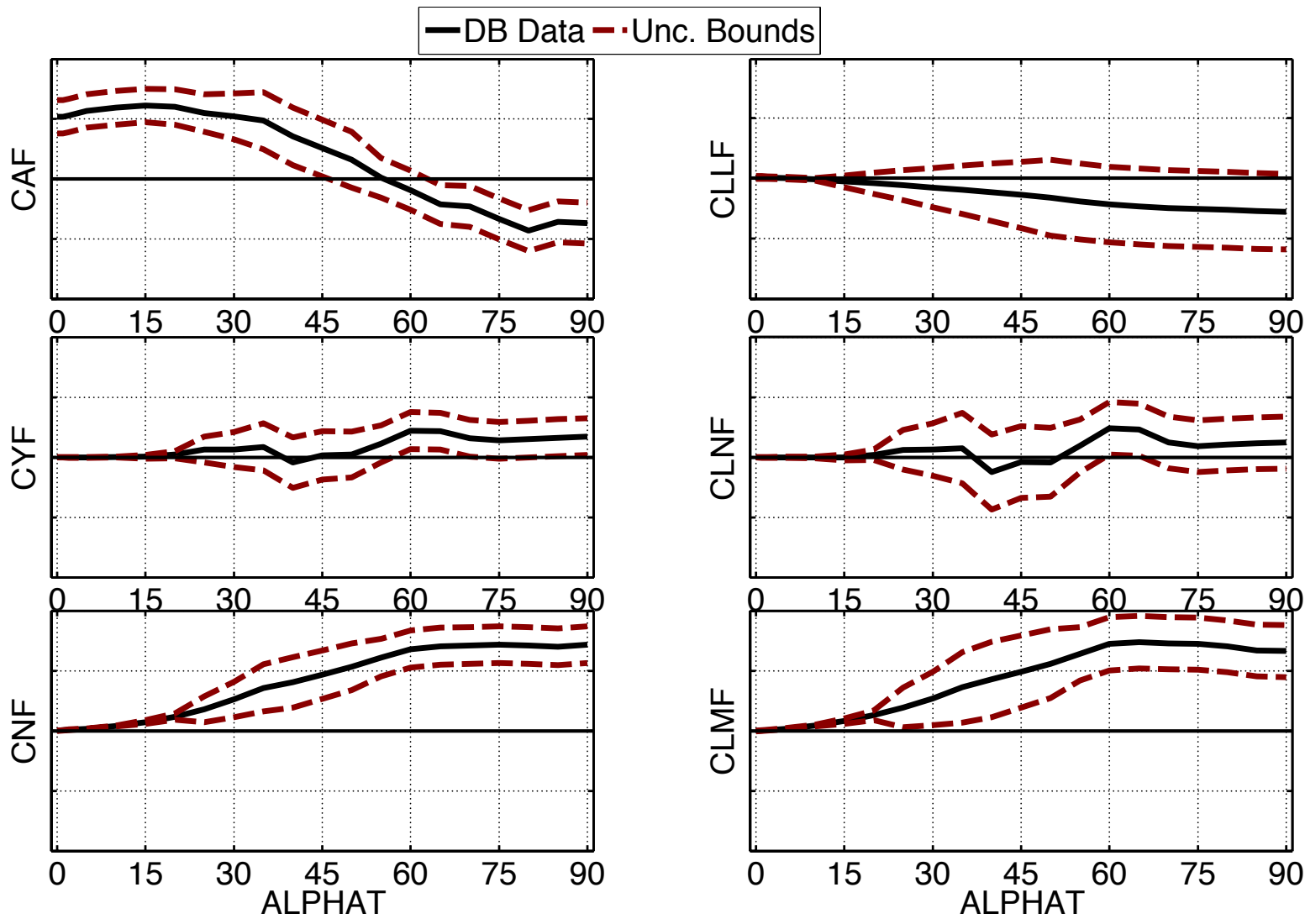

Figure 9. Transition DB and uncertainty bounds at $\phi_{a}=0$ degrees versus $\alpha_{T}$ (MRC at FS GP).

\section{B. Total Uncertainty for Tower Increments DB}

1. Total Uncertainty Buildup for Tower Increments DB

The Tower Increments DB total uncertainties were computed by substituting the results for each uncertainty source into Eq. (3). None of the uncertainty data varied significantly with any independent variable, so there is only a single uncertainty for each coefficient for the entire database domain. The effects of the tower were assumed to extend to $h / L$ of 1.2 based on WT data trends, and all coefficients and uncertainties in the Tower Increments DB were set to zero at $h / L$ of 1.2. Thus, the final Tower Increments DB uncertainties equal the values computed using Eq. (3) for all wind azimuth angles and $h / L$ values from 0.0 to 0.97 and then linearly decrease to zero at $h / L$ of 1.2. 
Figure 10 shows the three components of the uncertainty (experimental, database modeling, and database interpolation uncertainties) for the Tower Increments DB and the total uncertainty versus $h / L$. The experimental uncertainty $(U E X P)$ was by far the dominant component for all coefficients, while the database modeling uncertainty $(U D B M)$ and the database interpolation uncertainty $(U D B I)$ were not significant for the Tower Increments DB. Unlike for the Transition DB, no adjustments were made to the computed RSS values for the Tower Increments DB.

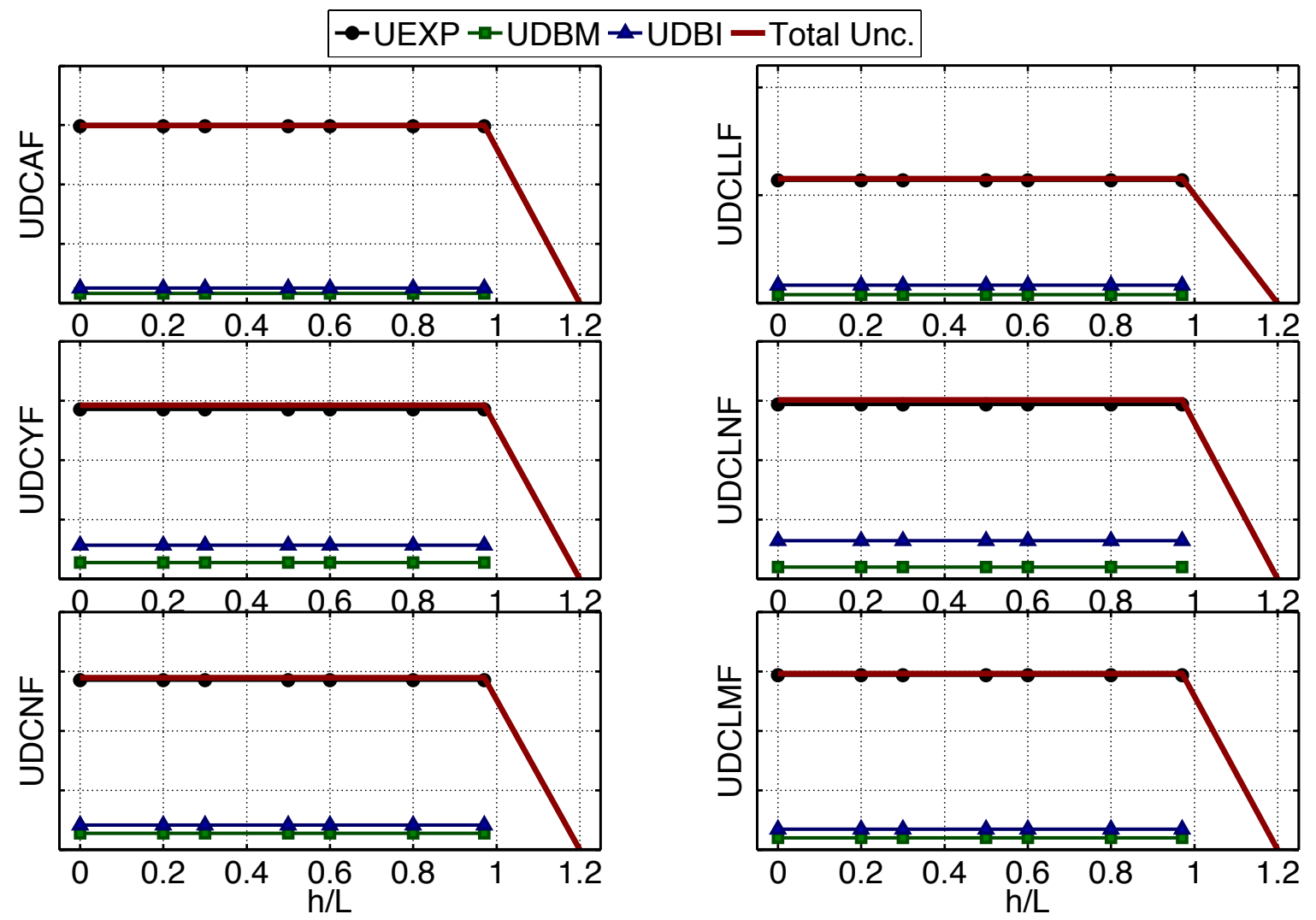

Figure 10. Tower Increments DB total uncertainty buildup and final uncertainties versus $h / L$.

\section{Tower Increments $D B$ and Uncertainty Visualization}

Figure 11 shows the database and maximum uncertainty bounds versus wind azimuth angle at $h / L$ of 0.0 . The database and uncertainties in the figure again use the MRC for the DB (FS GP) for $\Delta C_{m}$ and $\Delta C_{n}$. The uncertainties at the FS GP were computed by properly transferring the uncertainties to the FS GP. 

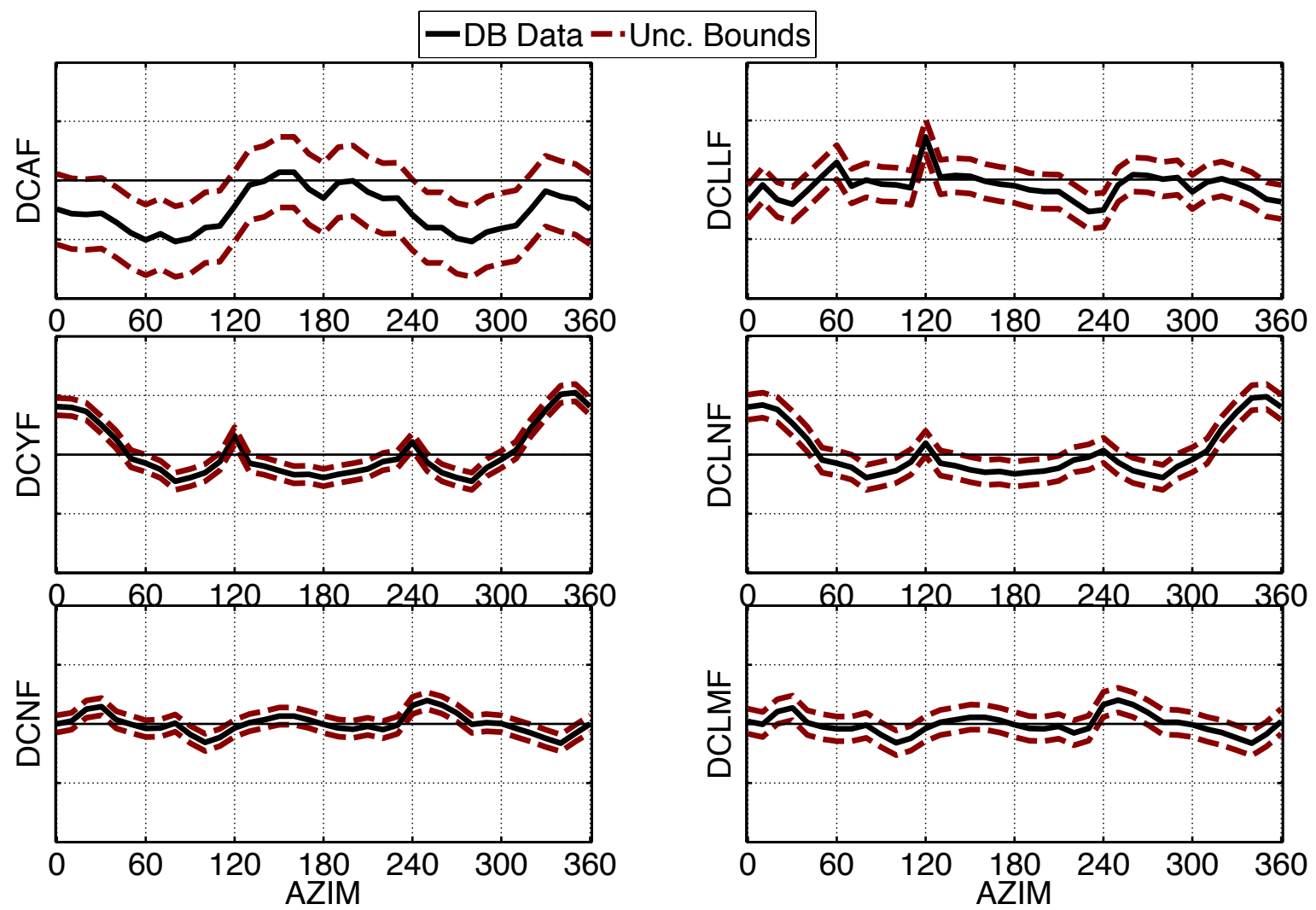

Figure 11. Tower Increments DB and uncertainty bounds at $h / L=0.0$ versus wind azimuth angle (MRC at FS GP).

\section{Uncertainties for Total A106 Liftoff/Transition DB}

Both segments of the A106 Liftoff/Transition DB must be used during simulations of the CLV after liftoff, since the Transition DB contains the freestream data and the Tower Increments DB contains the incremental effects of the launch tower. The crosswind dynamic pressure $\left(q_{\text {wind }}\right)$ associated with the wind incident on the tower assembly and neglecting any motion of the vehicle itself is used to compute the incremental forces and moments from the Tower Increments DB coefficients. These incremental forces and moments are then added to the freestream values computed using the total dynamic pressure $\left(q_{\infty}\right)$ and the Transition DB coefficients. Essentially, this assumes that the incremental effects of the tower are constant for any angle of attack and sideslip angle of the CLV. Since the total dynamic pressure increases relative to the crosswind dynamic pressure as the vehicle accelerates after liftoff, the contribution of the tower assembly interference (Tower Increments DB) becomes less significant, and the vehicle's freestream aerodynamics (Transition DB) dominate the aerodynamic force and moment values.

At the instant before liftoff, before the CLV begins moving relative to the tower assembly, so $q_{\infty}$ equals $q_{\text {wind }}$. For this condition (and only for this condition), the total aerodynamic coefficients for the CLV can be found by simply adding the Tower Increments DB values at $h / L$ of 0.0 to the Transition DB values at a total angle of attack of 90 degrees. At any moment after liftoff, the two dynamic pressures are different, and the total coefficient values cannot be computed directly. Figure 12 shows the total aerodynamic coefficients at the moment of liftoff versus wind azimuth angle, along with the total uncertainty bounds (MRC at the FS GP) and the freestream Transition DB data. The presence of the tower assembly reduces the magnitude of the side force coefficient for wind azimuth angles around 180 degrees, which corresponds to a wind from the south, and for wind azimuth angles from 315 degrees to 45 degrees, corresponding to northerly winds. A reduction in the side force coefficient for a south wind results in a smaller force pushing the CLV towards the launch tower. This is generally assumed to be a beneficial effect because it reduces the chance of contact with the tower after liftoff. 


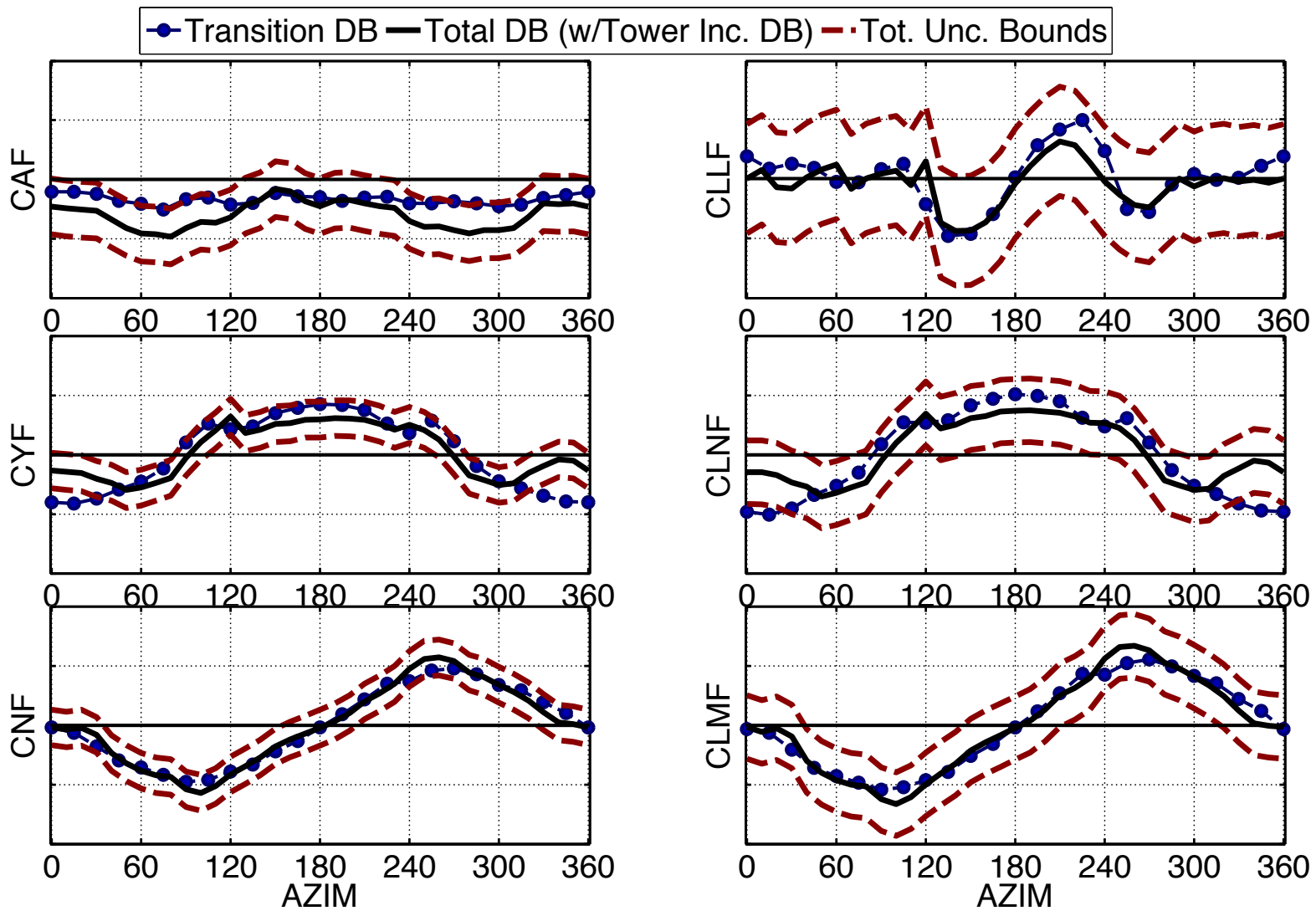

Figure 12. Total A106 Liftoff/Transition DB data and maximum uncertainty bounds at the instant before liftoff and Transition DB data at $\alpha_{T}=90$ degrees versus wind azimuth angle (MRC at FS GP).

\section{Comparisons with Previous Data}

Although the 14×22 T591 was the first experimental test of the Ares I CLV that covered the entire domain of the A106 Liftoff/Transition DB and included the effects of the tower assembly after liftoff, there were some earlier data from various sources to compare with the current database. An earlier database was developed for previous Ares I GN\&C analysis in this regime. This previous database was developed using engineering methods based on limited CFD data and empirical data. That database included three coefficients $\left(C_{N}, C_{A}\right.$, and $\left.C_{m}\right)$ in the missile axis system and did not include quantified uncertainties; instead, estimated dispersion bounds based on engineering judgment were provided. This earlier Ares I database and extensive additional CFD data on the Ares I-X Flight Test Vehicle (FTV) were used to construct the final preflight aerodynamic database for the Ares I-X FTV. The final Ares I-X FTV DB did not include any effects of the tower assembly. The Ares I-X FTV DB is representative of the aerodynamic data for the transition regime of flight used for Ares I GN\&C simulations prior to the release of the current A106 Liftoff/Transition DB.

Figure 13 shows the current A106 Transition DB and uncertainties compared to the Ares I-X FTV DB and uncertainties at Mach 0.3. Both databases are shown versus total angle of attack at an aerodynamic roll angle of 0 degrees, and the MRC for both databases is the FS GP. The Transition DB nominal data is the solid black line with square markers and the uncertainties are shown by the gray shaded region bounded by black dashed lines, while the Ares I-X FTV DB nominal data is the red line with circular markers and the corresponding uncertainties are shown by the pink shaded region bounded by dotted red lines. The figure shows that there is reasonable qualitative agreement for $C_{A}, C_{N}$, and $C_{m}$ between the Transition DB and the Ares I-X FTV DB. The plots for $C_{Y}$ and $C_{n}$ show some differences between the two databases, especially between 30 and 70 degrees. Closer agreement between the DBs for the longitudinal coefficients $\left(C_{A}, C_{N}\right.$, and $\left.C_{m}\right)$ than the lateral-directional coefficients $\left(C_{l}, C_{Y}\right.$, and $\left.C_{n}\right)$ at an aerodynamic roll angle of 0 degrees makes sense because the longitudinal coefficients in the missile axis system for the Ares I-X FTV DB were developed using higher fidelity data (CFD) than the lateral-directional coefficients 
(empirical data and engineering judgment). The A106 Transition DB uncertainties are significantly smaller in magnitude than the Ares I-X FTV DB uncertainties for $C_{N}$ and $C_{m}$ for total angles of attack above 60 degrees and throughout the domain for $C_{A}$. The $C_{l}$ data for both databases appear to agree well qualitatively at this condition, but examination of the data at other roll angles indicated significant differences between the DBs at most conditions. The current Transition DB uncertainties for $C_{l}$ are much larger than the uncertainties in the Ares I-X FTV DB.

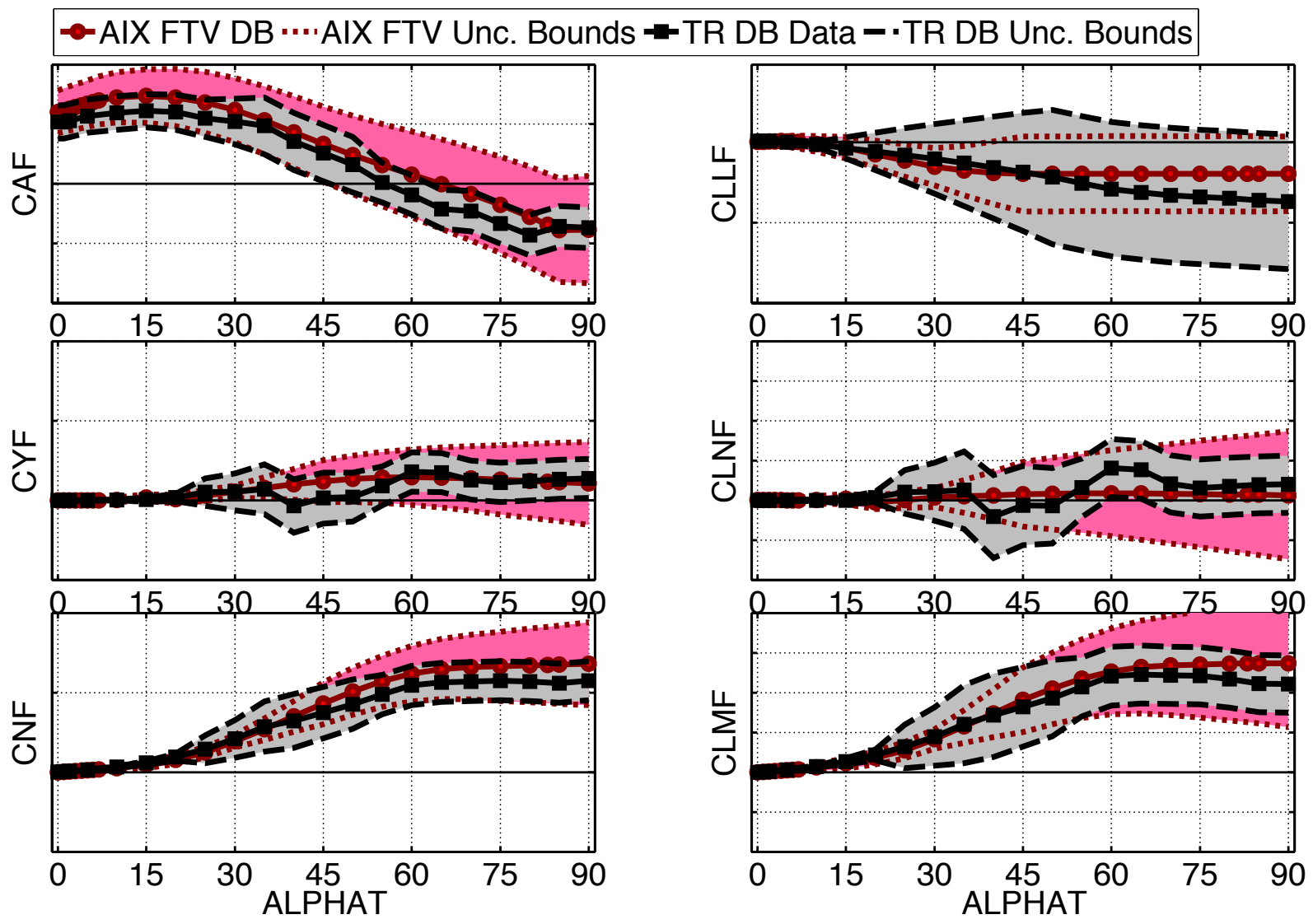

Figure 13. Comparison of current A106 Transition DB and uncertainty to the final Ares I-X FTV DB and uncertainty at $\phi_{a}=0$ degrees versus $\alpha_{T}$ (MRC at FS GP).

Figure 14 shows an expanded plot of the normal force at 0 degrees aerodynamic roll angle (the bottom left plot in Fig. 13). The normal force coefficient at this condition is generally representative of the primary loading on the CLV throughout the domain. The figure shows that the Transition DB nominal value at a total angle of attack of 90 degrees is about 20 percent less than the Ares I-X FTV DB value. In addition, the uncertainty interval at 90 degrees total angle of attack for the Transition DB is significantly smaller than the Ares I-X FTV DB uncertainty interval. The combination of these two factors results in about a 33 percent reduction in the maximum possible dispersed normal force coefficient for the Transition DB compared to the Ares I-X FTV DB. On the other hand, the Transition DB uncertainties are larger than the Ares I-X FTV DB uncertainties for total angles of attack between about 20 and 50 degrees. The WT test team noted significant flow dynamics in this regime during $14 \times 22$ T591, ${ }^{10}$ which supports data on similar bodies in previous literature. ${ }^{23}$ Therefore, the Transition DB uncertainties for the mid-range of total angle of attack reflect both the WT data and the knowledge from previous literature on similar bodies, while the Ares I-X FTV DB uncertainties may have been unrealistically low. 


\section{-AIX FTV DB …AIX FTV Unc. Bounds - TR DB Data - ·TR DB Unc. Bounds}

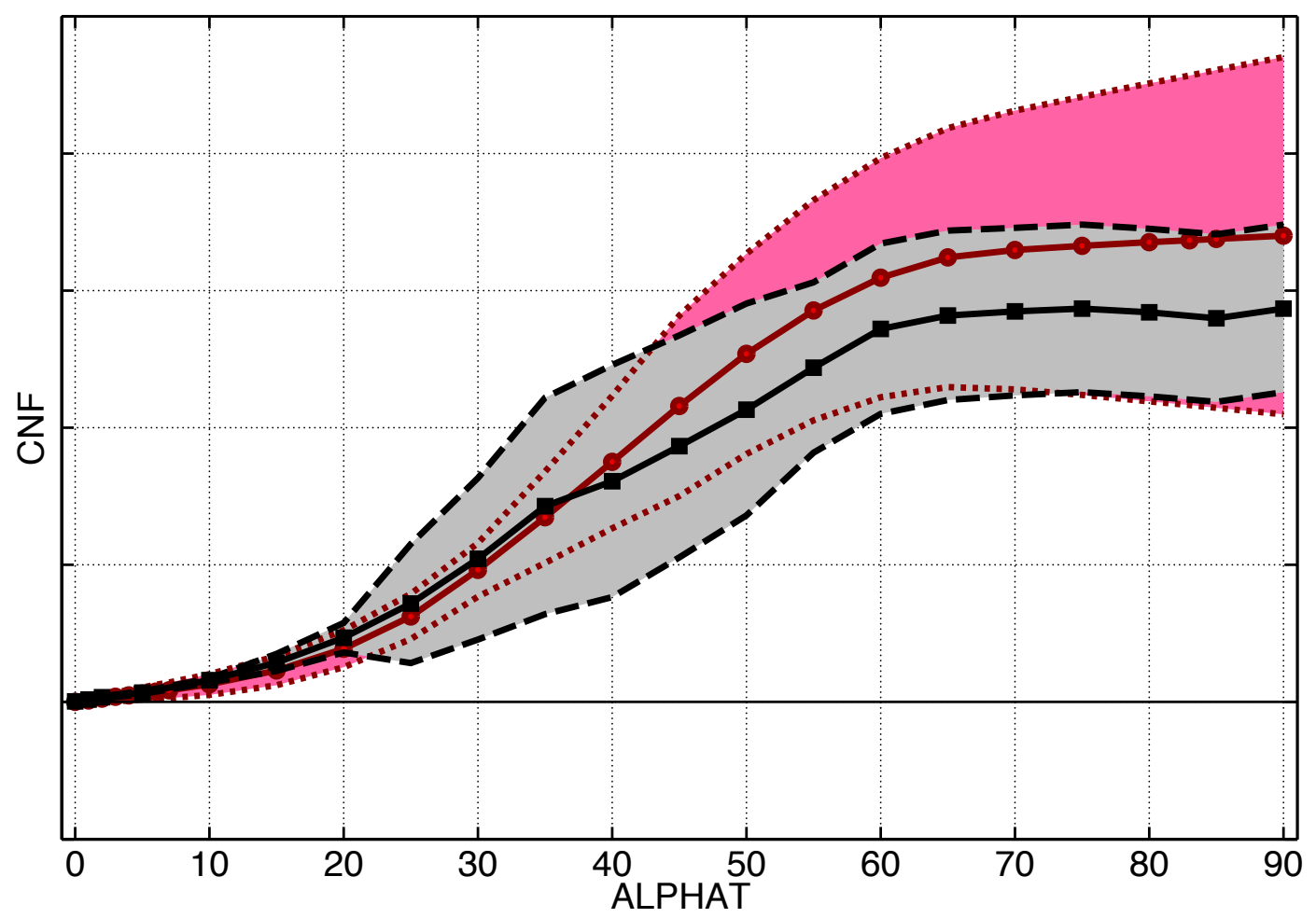

Figure 14. Comparison of $C_{N}$ data for current A106 Transition DB and uncertainty to the final Ares I-X FTV DB and uncertainty at $\phi_{a}=0$ degrees versus $\alpha_{T}$.

As noted above, previous databases did not account for the effect of the tower assembly on the aerodynamics of the vehicle after liftoff. Figure 15 compares the body axis system $C_{Y}$ from the A106 Liftoff/Transition DB to the Ares I-X FTV DB data at the moment of liftoff, when the total angle of attack equals 90 degrees and the total dynamic pressure equals the crosswind dynamic pressure. The data are plotted against wind azimuth angle, and the total $C_{Y}$ from the A106 Liftoff/Transition DB (Transition DB + Tower Increments DB) is the thick black line with square markers with the uncertainty interval shown as the gray shaded region bounded by dashed black lines, while the Ares I-X FTV DB is plotted as a red solid line with circular markers with the uncertainty interval shown as the pink shaded region bounded by the dotted red lines. The side force in this frame is of special interest during liftoff because positive values indicate forces pushing the vehicle towards the tower assembly. ${ }^{9,14}$ It is desirable to have the lowest possible side force magnitude in either direction, as large positive side forces could cause the vehicle to impact the tower, while large negative side forces could force the plume of the vehicle out of the blast zone, damaging the MLP. The largest positive $C_{Y}$ for the total DB occurs at a wind azimuth angle of 120 degrees. Figure 15 also shows that the magnitudes of both the total database and the maximum dispersed values possible within the uncertainty intervals for the A106 Liftoff/Transition DB are lower than the maximum possible magnitudes for the Ares I-X FTV DB for most of the wind azimuth angle domain. There is approximately a 40 percent reduction in the maximum possible positive $C_{Y}$ compared to the Ares I-X FTV DB. This reduction is caused partially by the reduction in the freestream data (Transition DB) as shown above in Fig. 14 and partially due to the effects of the tower assembly included in the A106 Liftoff/Transition DB, which were not modeled in the Ares I-X FTV DB. The effects of the tower assembly are especially noticeable for wind azimuth angles from 330 to 30 degrees and 120 to 240 degrees (see Ref. 9 and 14 for more discussion of the tower effects). 


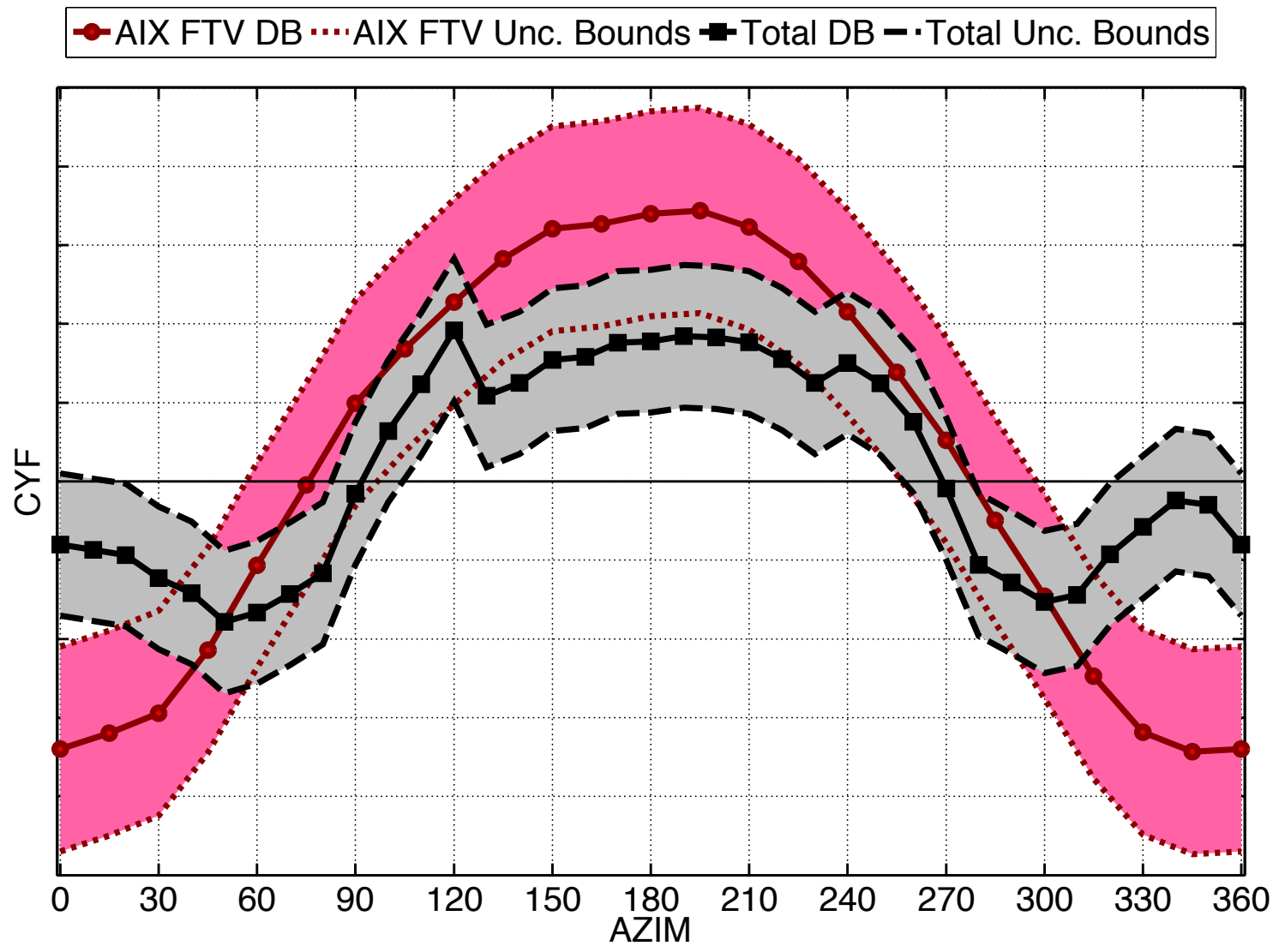

Figure 15. Comparison of total A106 Liftoff/Transition DB $C_{Y}$ data and uncertainty at the moment of liftoff to the final Ares I-X FTV DB and uncertainty versus wind azimuth angle.

\section{Conclusion}

This report detailed the entire uncertainty quantification process for the A106 Liftoff/Transition DB. The methodology used was similar to the UQ analyses for other recent Ares I aerodynamic databases and involved identifying and estimating uncertainties for all major error sources during data acquisition, database development, and database implementation. The experimental uncertainty in the $14 \times 22$ T591 data was the most significant source of uncertainty for both segments of the A106 Liftoff/Transition DB. This database and accompanying uncertainty models represent a significant improvement in the fidelity of the aerodynamic data available to the Ares I project in the flight regime from liftoff through transition to flight at low total angles of attack. This paper also compared the database and uncertainties to previous databases and uncertainties developed for the Ares I CLV and the Ares I-X FTV. The resulting comparisons suggest that the changes in the database and uncertainties represented in this release may have a significant impact on GN\&C simulations of the CLV flight.

\section{Acknowledgments}

The author would like to thank Amber L. Favaregh for performing the experimental repeatability computations and Jeremy Pinier for his extensive independent verification and review of the UQ methodology. Also, Mike Hemsch, Heather Houlden, and the rest of the Ares and Orion UQ teams deserve credit for their aid in the development of the overall UQ methodology. Finally, the author greatly appreciated Kay Forrest's editorial help in preparing this manuscript.

\section{References}

${ }^{1}$ Hanke, J. L., "Uncertainty Quantification and Modeling for the Ares I A106 Liftoff/Transition Aerodynamic Database," NASA Ares-AD-TA-0016, April 2011. 
${ }^{2}$ Hall, R. M., Holland, S. D., and Blevins, J. A., “Aerodynamic Characterization Overview of a Modern Launch Vehicle," AIAA-2011-10, January 2011.

${ }^{3}$ Tomek, W. G., Erickson, G. E., Pinier, J. T., and Hanke, J. L., "Overview of Experimental Investigations for Ares I Launch Vehicle Development," AIAA-2011-13, January 2011.

${ }^{4}$ Pinier, J. T., Niskey, C. J., Hanke, J. L., and Tomek, W. G., “Ares I Aerodynamic Testing at the Boeing Polysonic Wind Tunnel," AIAA-2011-998, January 2011.

${ }^{5}$ Abdol-Hamid, K. S., Ghaffari, F. and Parlette, E. B., "Overview of Ares-I CFD Ascent Aerodynamic Data Development And Analysis Based on USM3D," AIAA-2011-15, January 2011.

${ }^{6}$ Deere, K. A., Pao, S. P., and Abdol-Hamid, K. S., "Computational Analysis of Ares I Roll Control System Jet Interaction Effects on Rolling Moment," AIAA-2011-172, January 2011.

${ }^{7}$ Pandya, M. J., Frink, N. T., Abdol-Hamid, K. S., Samareh, J. A., Parlette, E. B., and Taft, J. T., "Enhancements to NASA TetrUSS for Constellation Program," AIAA-2011-1111, January 2011.

${ }^{8}$ Pamadi, B. N., Pei, J., Pinier, J. T., Klopfer, G. H., Holland, S. D., and Covell, P. F., "Aerodynamic Analyses and Database Development for Ares I Vehicle First Stage Separation,” AIAA-2011-171, January 2011.

${ }^{9}$ Capone, F. J., Paulson, J. W. Jr., and Erickson, G. E., "Liftoff and Transition Aerodynamics of the Ares I (A106) Launch Vehicle," AIAA-2011-997, January 2011.

${ }^{10}$ Hall, R. M., and Banks D. W., "Progress in Developing Gritting Techniques for High Angle of Attack Flows," AIAA-940169, Jan. 1994.

${ }^{11}$ Hall, R. M., Erickson, G. E., Fox, C. H., Jr., Banks, D. W., and Fisher, D. F., "Evaluation of Gritting Strategies for High Angle of Attack Using Wind Tunnel and Flight Test Data for the F/A-18," NASA TP-1998-207670, May 1998.

${ }^{12}$ Braslow, A. L., Harris, R. V., Jr., and Hicks, R. M., "Use of Grit-Type Boundary-Layer Transition Strips on Wind-Tunnel Models,"NASA TN D-3579, 1966.

${ }^{13}$ Crew Launch Vehicle Mobile Launcher, Launch Complex 39 Design Criteria. Drawing Number 242MDC00001, NASA Kennedy Space Center, Dec. 2008.

${ }^{14}$ Pamadi, B. N., Pei, J., Covell, P. F., Favaregh, N. M., Gumbert, C. G., and Hanke, J. L., “Aerodynamic Analyses and Database Development for Lift-Off/Transition and First Stage Ascent of the Ares I A106 Vehicle," AIAA-2011-12, January 2011.

${ }^{15}$ Houlden, H. P, Favaregh, A. L., and Hemsch, M. J., "Quantification of the Uncertainties for the Ares I A106 Ascent Aerodynamic Database,” AIAA-2010-4926, June 2010.

${ }^{16}$ Hemsch, M. J, and Walker, E. L., "The Crucial Role of Error Correlation for Uncertainty Modeling of CFD-Based Aero Increments," To be presented at the $49^{\text {th }}$ AIAA Aerospace Sciences Meeting, Orlando 2011.

${ }^{17}$ Coleman, H. W., and Steele, W. G., Experimentation and Uncertainty Analysis for Engineers, $2^{\text {nd }}$ ed., John Wiley \& Sons, Inc., New York, NY, 1999.

${ }^{18}$ Wheeler, D. J., Understanding Industrial Experimentation, SPC Press Inc, Knoxville, TN, 1990.

${ }^{19}$ Owen, D. B., Handbook of Statistical Tables, Addison-Wesley Publication Company, Inc, Reading, MA, 1962.

${ }^{20}$ Hemsch, M. J., Hanke, J. L., Walker, E. L., and Houlden, H. P., "Detailed Uncertainty Analysis for Ares I Ascent Aerodynamics Wind Tunnel Database," AIAA-2008-4259, June 2008.

${ }^{21}$ Hemsch, M. J., "Characterization of Within-Test Repeatability for LaRC UPWT Tests 1813 and 1968 (Ares I ADAC2A)," NASA Ares-AD-TA-0006, August 20, 2007.

${ }^{22}$ U.S. Guide to Expression of Uncertainty in Measurement, American National Standards Institute, ANSI/NCSL Z540.21997 (R2002).

${ }^{23}$ Polhamus, E. D., “A Review of Some Reynolds Number Effects Related to Bodies at High Angles of Attack,” NASA CR 3809, August 1984

${ }^{24}$ Coleman, H. W., and Steele, W. G., Experimentation and Uncertainty Analysis for Engineers, $2^{\text {nd }}$ ed., John Wiley \& Sons, Inc., New York, NY, 1999. 